\title{
The Trypanosome UDP-Glucose Pyrophosphorylase Is Imported by Piggybacking into Glycosomes, Where Unconventional Sugar Nucleotide Synthesis Takes Place
}

\author{
Oriana Villafraz, ${ }^{a}$ Hélène Baudouin, a,b Muriel Mazet, ${ }^{a, b}$ Hanna Kulyk, ${ }^{c, d}$ Jean-William Dupuy, Erika Pineda, ${ }^{a}$ (D) Cyrille Botté, ${ }^{f}$

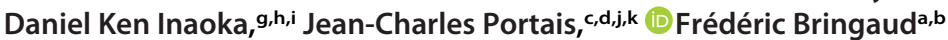 \\ aUniversité de Bordeaux CNRS, Microbiologie Fondamentale et Pathogénicité (MFP), UMR 5234, Bordeaux, France \\ bUniversité de Bordeaux CNRS, Centre de Résonance Magnétique des Systèmes Biologiques (CRMSB), UMR 5536, Bordeaux, France \\ cToulouse Biotechnology Institute (TBI), INSA de Toulouse INSA/CNRS 5504, UMR INSA/INRA, Toulouse, France \\ dMetaboHUB-MetaToul, National Infrastructure of Metabolomics and Fluxomics, Toulouse, France \\ eUniversité de Bordeaux Plateforme Protéome, Bordeaux, France \\ fApicolipid Team, Institute for Advanced Biosciences, CNRS UMR5309, INSERM U1209, Université Grenoble Alpes, La Tronche, France \\ gDepartment of Biomedical Chemistry, Graduate School of Medicine, The University of Tokyo, Tokyo, Japan \\ hSchool of Tropical Medicine and Global Health, Nagasaki University, Nagasaki, Japan \\ 'Department of Molecular Infection Dynamics, Shionogi Global Infectious Disease Division, Institute of Tropical Medicine (NEKKEN), Nagasaki University, Nagasaki, Japan \\ jRESTORE, Université de Toulouse, INSERM U1031, CNRS 5070, Toulouse, France \\ kEFS, Université Paul Sabatier, Toulouse, France
}

ABSTRACT Glycosomes are peroxisome-related organelles of trypanosomatid parasites containing metabolic pathways, such as glycolysis and biosynthesis of sugar nucleotides, usually present in the cytosol of other eukaryotes. UDP-glucose pyrophosphorylase (UGP), the enzyme responsible for the synthesis of the sugar nucleotide UDP-glucose, is localized in the cytosol and glycosomes of the bloodstream and procyclic trypanosomes, despite the absence of any known peroxisome-targeting signal (PTS1 and PTS2). The questions that we address here are (i) is the unusual glycosomal biosynthetic pathway of sugar nucleotides functional and (ii) how is the PTS-free UGP imported into glycosomes? We showed that UGP is imported into glycosomes by piggybacking on the glycosomal PTS1-containing phosphoenolpyruvate carboxykinase (PEPCK) and identified the domains involved in the UGP/PEPCK interaction. Proximity ligation assays revealed that this interaction occurs in 3 to $10 \%$ of glycosomes, suggesting that these correspond to organelles competent for protein import. We also showed that UGP is essential for the growth of trypanosomes and that both the glycosomal and cytosolic metabolic pathways involving UGP are functional, since the lethality of the knockdown UGP mutant cell line ( ${ }^{R N A}$ UGP, where RNAi indicates RNA interference) was rescued by expressing a recoded UGP (rUGP) in the organelle ( ${ }^{R N A i} U G P /{ }^{E X P}$ rUGP-GPDH, where GPDH is glycerol-3-phosphate dehydrogenase). Our conclusion was supported by targeted metabolomic analyses (ion chromatography-high-resolution mass spectrometry [IC-HRMS]) showing that UDPglucose is no longer detectable in the RNAiUGP mutant, while it is still produced in cells expressing UGP exclusively in the cytosol (PEPCK null mutant) or glycosomes (RNAIUGP/EXP rUGP-GPDH). Trypanosomatids are the only known organisms to have selected functional peroxisomal (glycosomal) sugar nucleotide biosynthetic pathways in addition to the canonical cytosolic ones.

IMPORTANCE Unusual compartmentalization of metabolic pathways within organelles is one of the most enigmatic features of trypanosomatids. These unicellular eukaryotes are the only organisms that sequestered glycolysis inside peroxisomes (glycosomes), although the selective advantage of this compartmentalization is still not
Citation Villafraz O, Baudouin $\mathrm{H}$, Mazet $\mathrm{M}$, Kulyk H, Dupuy J-W, Pineda E, Botté C, Inaoka DK, Portais J-C, Bringaud F. 2021. The trypanosome UDP-glucose pyrophosphorylase is imported by piggybacking into glycosomes, where unconventional sugar nucleotide synthesis takes place. mBio 12:e00375-21. https://doi.org/10.1128/mBio.00375-21. Editor Dominique Soldati-Favre, University of Geneva

Copyright $\odot 2021$ Villafraz et al. This is an open-access article distributed under the terms of the Creative Commons Attribution 4.0 International license. Address correspondence to Frédéric Bringaud, frederic.bringaud@u-bordeaux.fr.

Received 16 February 2021

Accepted 5 April 2021

Published 28 May 2021 
clear. Trypanosomatids are also unique for the glycosomal localization of enzymes of the sugar nucleotide biosynthetic pathways, which are also present in the cytosol. Here, we showed that the cytosolic and glycosomal pathways are functional. As in all other eukaryotes, the cytosolic pathways feed glycosylation reactions; however, the role of the duplicated glycosomal pathways is currently unknown. We also showed that one of these enzymes (UGP) is imported into glycosomes by piggybacking on another glycosomal enzyme (PEPCK); they are not functionally related. The UGP/PEPCK association is unique since all piggybacking examples reported to date involve functionally related interacting partners, which broadens the possible combinations of carrier-cargo proteins being imported as hetero-oligomers.

KEYWORDS Trypanosoma brucei, UDP-glucose pyrophosphorylase, glycosomes, peroxisomes, piggybacking, procyclic form

7 rypanosoma brucei is a parasite responsible for human African trypanosomiasis, also known as sleeping sickness, a disease affecting sub-Saharan Africa that can be fatal if left untreated (1). This parasite is transmitted through the bite of a tsetse fly and has a complex developmental cycle, including the bloodstream form (BSF) and the procyclic form (PCF) found in the blood of mammalian hosts and the digestive tract of the insect, respectively. A major difference between these two forms is their modes of energy conservation, with the former depending on glucose via glycolysis and the latter being able to use glucose, proline, and other amino acids as carbon sources (2). The complexity of $T$. brucei's life cycle leads to the capacity for fast and high adaptation to environmental conditions, mostly through metabolic changes related to energy metabolism. One of the factors playing a role in these efficient changes is the presence of peroxisome-related organelles called glycosomes. The glycosomes contain the first six or seven glycolytic steps, which are commonly present in the cytosol of other eukaryotic cells (3). In addition, the glycosomes contain up to a dozen other metabolic pathways, including the sugar nucleotide biosynthetic pathways, which are also exclusively cytosolic in other organisms (4).

All eukaryotes, excepted trypanosomatids, synthesize sugar nucleotides in the cytosol and then transport them into the lumen of the endoplasmic reticulum (ER) or Golgi apparatus to feed glycosyltransferase-dependent glycosylation reactions (5). In the particular case of trypanosomatids, most of the enzymes involved in de novo biosynthesis of sugar nucleotides are present in the glycosomes (6-11). Some of them are known to be essential for the parasite's survival, probably because the cell surface and endoso$\mathrm{mal} /$ lysosomal systems are rich in essential glycoconjugates (12).

Within the steps involved in the production of sugar nucleotides, UDP-glucose pyrophosphorylase (UGP) catalyzes the coupling of glucose 1-phosphate (G1P) and UTP to produce UDP-glucose (UDP-GIc) (13). UDP-Glc is a central metabolite that acts as a glucose donor in several pathways, as exemplified by UDP-Glc:glycoprotein glucosyltransferase (UGGT), which uses this sugar nucleotide as a glucosyl donor for protein glycosylation. UDP-Glc has an important role in glycoprotein quality control in the ER, because UGGT specifically glycosylates unfolded glycoproteins to prevent their processing toward the cytosol (14). UDP-Glc is also the obligate precursor of UDP-galactose (UDP-Gal) via a reaction catalyzed by UDP-Glc 4'-epimerase (GalE), given that the parasite hexose transporters are unable to transport galactose (15). The lethality of the $T$. brucei GalE null mutant makes UDP-Glc production essential for the parasite (9). In the closely related parasites Trypanosoma cruzi and Leishmania major, UDP-sugar pyrophosphorylase (USP) can also activate G1P, in addition to galactose 1-phosphate, while the $T$. brucei genome does not contain the USP-orthologous gene. Consequently, the simultaneous deletion of the USP and UGP genes is required to deplete the Leishmania cells of UDP-Glc and UDP-Gal, leading to growth arrest and cell death (16). In contrast to the animal and fungal UGP, which are octameric (17) and can be regulated by redox mechanisms (18-20) or phosphorylation (21), the characterized T. brucei and L. major UGPs are active as monomers and are regulated by allosteric mechanisms $(7,17,22)$. 
As recently shown for most of the T. brucei enzymes involved in the biosynthesis of sugar nucleotides, the $T$. brucei UGP was reported to be localized in glycosomes of BSFs $(7,23)$. However, it does not contain any of the canonical peroxisomal targeting signals (PTSs) required for import of proteins into the organelle, i.e., the PTS1 tripeptide ([STAGCN]-[RKH]-[LIVMAFY]) or PTS2 ([M]- $\mathrm{X}_{0 / 20}-[\mathrm{RK}]-[\mathrm{LVI}]-\mathrm{X}_{5}-[\mathrm{HQ}]-[\mathrm{ILAF}]$, where $\mathrm{X}$ refers to any amino acid [with its number in subscript]) located at the $\mathrm{C}$ - and $\mathrm{N}$-terminal extremities of the peroxisomal/glycosomal proteins, respectively (24). Alternatively, proteins lacking a PTS can be imported into the organelle by piggybacking through interaction with a PTS-containing protein. The very few examples of piggybacking described so far in peroxisomes of mammals $(25,26)$, plants $(27)$, and Saccharomyces cerevisiae $(28,29)$ involve hetero-oligomeric complexes formed by protein isoforms or by functionally related proteins. This mechanism of import has been proposed as an explanation for the presence of some PTS-lacking proteins within glycosomes but has not yet been reported in trypanosomatids so far.

Here, we showed that UGP is imported into glycosomes by interacting with the glycosomal PTS1-containing phosphoenolpyruvate carboxykinase (PEPCK), supporting coimport of functionally unrelated proteins. We also showed that UGP is an essential enzyme for the growth of trypanosomes with dual cytosolic and glycosomal localizations. Metabolomic analyses revealed that UDP-Glc is produced by functional cytosolic and glycosomal pathways. The positive selection of functional sugar nucleotide biosynthesis within glycosomes of trypanosomatids, while this pathway is exclusively cytosolic in other eukaryotes, raises questions about its role in these parasites.

\section{RESULTS}

UDP-glucose pyrophosphorylase (UGP) has dual glycosomal and cytosolic localizations. Previous studies on the UGP subcellular localization revealed that the protein is associated with glycosomes of the BSF (7), despite the absence of any predicted peroxisomal targeting signal (PTS1/PTS2). We raised an anti-UGP ( $\alpha$ UGP) immune serum to confirm this unique glycosomal localization of UGP in the PCF by Western blotting of glycosomal and cytosolic fractions prepared by differential centrifugation, using control antibodies against glycosomal (NADH-dependent fumarate reductase [FRDg]) and cytosolic (enolase [ENO]) proteins. The anti-UGP immune serum detected a 55-kDa protein corresponding to the predicted size of UGP (theoretical molecular weight [MW], $54.5 \mathrm{kDa}$ ) in both the glycosomal and cytosolic fractions (Fig. 1A). This dual localization was further confirmed by digitonin titration, as UGP was released together with the cytosolic protein at low concentrations of detergent and the UGP signal increased with the digitonin concentration required to release the glycosomal marker (Fig. 1B). The increased signal at higher digitonin concentrations suggests that the total amount of UGP in the glycosomes is at least equivalent to that in the cytosol. We also addressed the UGP subcellular localization in BSFs by performing hypotonic lysis, which released cytosolic proteins, while glycosomal proteins remained in the cellular pellet, as evidenced by the glycosomal aldolase and cytosolic enolase markers (Fig. 1C). UGP is similarly distributed over the two compartments in BSFs, as observed for PCFs (Fig. 1C).

PEPCK-dependent import of UGP into glycosomes. Incidentally, a comparative proteomic analysis of the previously obtained PEPCK null ( $\Delta$ pepck) mutant (30) and the parental cell line, carried out in order to control the PEPCK gene deletion, showed a strong reduction (19.7-fold) of UGP peptide counts in the enriched glycosomal fractions of the mutant (see the PXD020190 data set in the PRIDE partner repository). Depletion of UGP in the glycosomes of the $\Delta$ pepck cell line was confirmed by Western blotting, showing that UGP was no longer detected in the $\Delta$ pepck glycosomes, while the protein was still present in the total cell extracts (Fig. 1D). Importantly, reexpression of the PEPCK gene in the PEPCK null background ( $\triangle$ pepck/EXPPEPCK.i cell line ["EXP" stands for "expressing," and ".i" stands for tetracycline-induced]) rescued the glycosomal localization of UGP (Fig. 1D). These data suggest that import of UGP into the glycosomes depends on the presence of PTS1-containing PEPCK, potentially by the so-called 

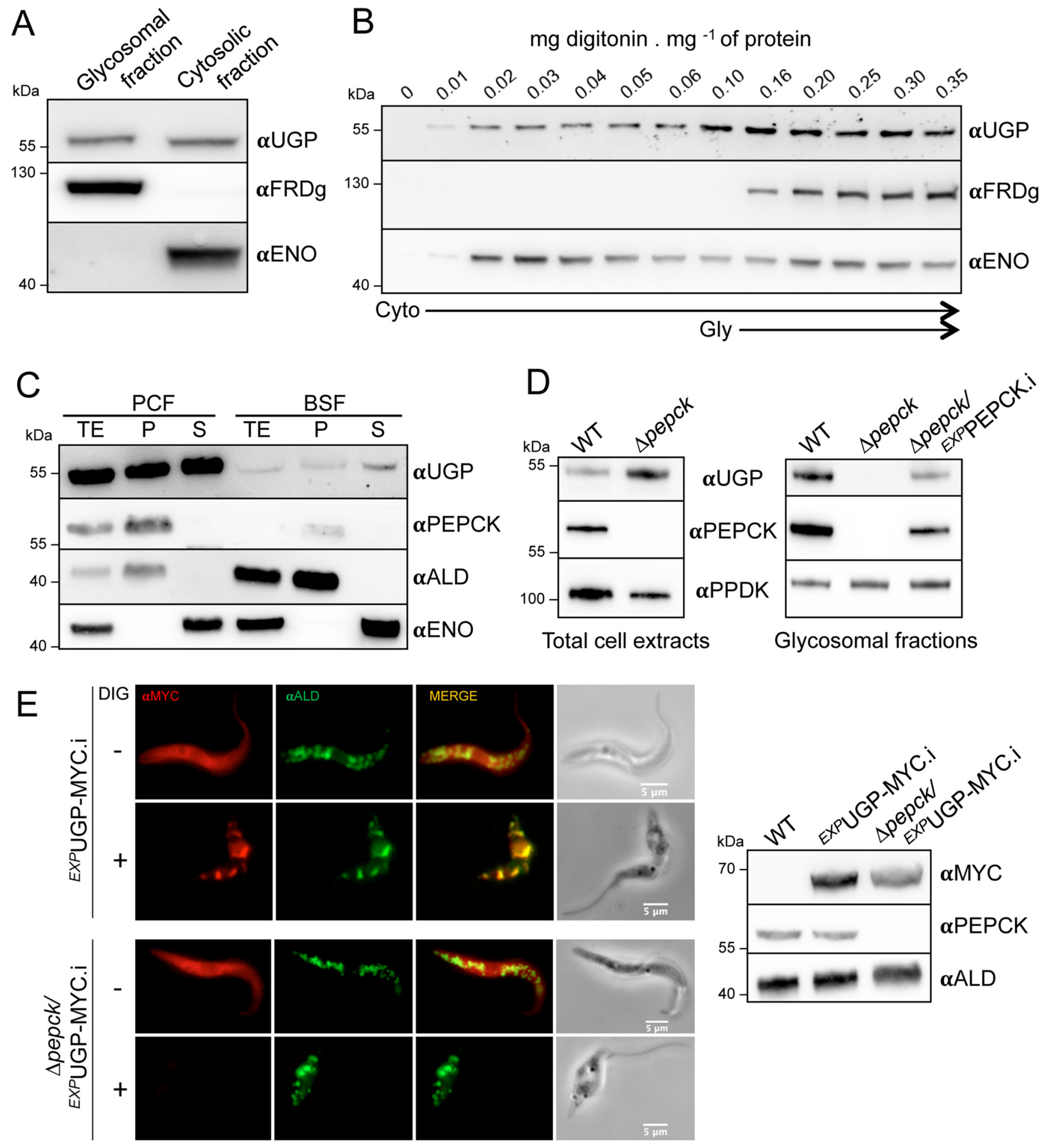

FIG 1 UGP has a dual localization in PCF and BSF, and its import into glycosomes depends on the PTS1-cointaining protein PEPCK. (A and B) Subcellular localization of UGP in the EATRO1125.T7T procyclic trypanosomes. (A) Enriched glycosomal and cytosolic fractions were obtained by differential centrifugation and analyzed by Western blotting using the anti-UGP antibodies ( $\alpha$ UGP), as well as immune sera against the glycosomal NADH-dependent fumarate reductase $(\alpha \mathrm{FRDg})$ and cytosolic enolase ( $\alpha \mathrm{ENO}$ ) markers. (B) UGP localization was also studied by digitonin titration. The supernatants collected from the parental cells incubated with 0 to $0.35 \mathrm{mg}$ of digitonin per $\mathrm{mg}$ of protein were analyzed by Western blotting using the immune sera indicated in the left margin. (C) Comparison of the subcellular localizations and protein expression levels of UGP and PEPCK, as well as the aldolase glycosomal and enolase cytosolic markers, in PCF and BSF trypanosomes. Total extracts (TE), pellets (P), and supernatants (S) obtained after hypotonic lysis were analyzed by Western blotting using the immune sera indicated. (D) Western blot analysis of total cellular extracts and glycosomal fractions of the WT, the $\Delta p e p c k$

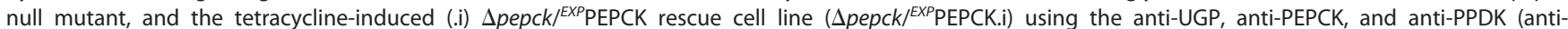
pyruvate phosphate dikinase) immune sera. (E, left) the UGP subcellular localization was analyzed by immunofluorescence of cell lines expressing a 
piggybacking mechanism not reported so far in trypanosomatids (31). In this context, UGP would be cotransported with PEPCK, which is imported into the glycosome via its PTS1.

To confirm the dual subcellular localization of UGP, we produced cell lines expressing a MYC-tagged UGP under the control of tetracycline in both the parental and the $\Delta$ pepck backgrounds (Fig. 1E, right panel). Immunofluorescence analyses showed a clear cytosolic pattern in the tetracycline-induced EXPUGP-MYC.i and $\Delta$ pepck/EXPUGPMYC.i cell lines (Fig. 1E, left panel). A signal colocalizing with the glycosomal marker aldolase was detected for the EXPUGP-MYC.i cells only after pretreatment with $0.04 \mathrm{mg}$ of digitonin per $\mathrm{mg}$ of protein required for permeabilization of the plasma membrane. These data confirmed that recombinant UGP-MYC exhibits dual localizations, similar to that in the native protein. Interestingly, the glycosomal signal was not detected in the $\Delta$ pepck/UGP-MYC.i cell line after digitonin treatment, indicating that all UGP localizes exclusively in the cytosol of this mutant. Altogether, these data support the role of PEPCK in the import of UGP into glycosomes.

UGP interacts with PEPCK in some glycosomes. To evidence the putative interaction between UGP and PEPCK, we used proximity ligation assays (PLA; Duolink), which enable detection of protein interactions, including transient/weak interactions in situ, with high specificity and sensitivity (32). We produced a $\triangle$ pepck/EXPTY-PEPCK/EXPUGPMYC cell line expressing TY-tagged PEPCK (TY-PEPCK; TY stands for the Ty1 epitope: EVHTNQDPLD) and MYC-tagged UGP (UGP-MYC) in the PEPCK null background. Briefly, the second PEPCK allele of the single-allele $\triangle$ pepck/PEPCK knockout cell line was replaced by a TY-PEPCK copy encoding TY-PEPCK tagged at its $\mathrm{N}$-terminal extremity to preserve the PTS1 motif required for glycosomal import. This $\triangle$ pepck/EXPTY-PEPCK cell line was transfected with the pLew100-EXPUGP-MYC plasmid to express UGP-MYC under the control of tetracycline. As controls, the UGP-MYC and TY-PEPCK recombinant proteins have been independently expressed in the $\Delta$ pepck ( $\triangle$ pepck/EXPUGP-MYC) and parental (EXPTY-PEPCK) backgrounds, respectively. The expression of both recombinant proteins, the specificity of the primary antibodies, and the glycosomal import of TYPEPCK and UGP-MYC were confirmed by Western blotting analyses of enriched glycosomal and cytosolic fractions, digitonin titration, and immunofluorescence analyses (see Fig. S1 in the supplemental material). As expected, TY-PEPCK showed a glycosomal localization; however, its level of expression was $\sim 8$ times lower than that of the native protein (Fig. S1A, compare the upper and lower bands of the $\alpha$ PEPCK signal in the EXPTY-PEPCK cell line, respectively). Despite this difference in expression levels, a significant part of the recombinant UGP-MYC is imported into the glycosomes of the tetracycline-induced $\Delta$ pepck/EXPTY-PEPCK/EXPUGP-MYC.i cell line (Fig. S1A and B), while remaining exclusively in the cytosol of the $\triangle$ pepck/EXPUGP-MYC.i cell line (Fig. S1A), as previously shown (Fig. 1E). $\alpha \mathrm{MYC}$ (rabbit) and $\alpha \mathrm{TY}$ (mouse) were validated to be specific and sensitive enough for us to perform PLA analysis (Fig. S1C).

PLA-positive puncta (red signals) corresponding to TY-PEPCK/UGP-MYC heterooligomers were observed in $62 \%$ of the $\triangle$ pepck/EXPTY-PEPCK/EXPUGP-MYC.i cells, while only $7 \%$ and $6 \%$ of the control $\triangle$ pepck/EXPUGP-MYC.i and EXPTY-PEPCK.i cells were positive, respectively (Fig. $2 \mathrm{~A}$ and $\mathrm{B}$ ). In addition, $\sim 90 \%$ of the positive $\Delta$ pepck/EXPUGPMYC.i and EXPTY-PEPCK.i cells contained a single dot, and the other $10 \%$ contained 2 dots, while the number of dots per cell in the $\Delta$ pepck/EXPTY-PEPCK/EXPUGP-MYC.i population was much higher, with $62 \%$ of the cells showing 2 to 10 dots (Fig. 2B). These data are in agreement with interactions between UGP-MYC and TY-PEPCK in $\Delta$ pepck/EXPTY-PEPCK/EXPUGP-MYC.i cells, while the very few red dots observed within the control $\Delta$ pepck/EXPUGP-MYC.i and EXPTY-PEPCK.i cells represent background signals.

FIG 1 Legend (Continued)

recombinant MYC-tagged UGP in the WT (EXPUGP-MYC cell line) and $\Delta$ pepck ( $\Delta$ pepck/EXP UGP-MYC) backgrounds, using anti-MYC (red) and the glycosomal ALD (green) control. Before fixation, the cells were pretreated with $0.04 \mathrm{mg}$ digitonin (DIG)/mg of protein (+) to remove the cytosolic UGP-MYC signal or not treated (-). The expression of UGP-MYC was confirmed by Western blotting of total cell extracts (right) using anti-MYC, anti-PEPCK, and anti-ALD as loading controls. 
A

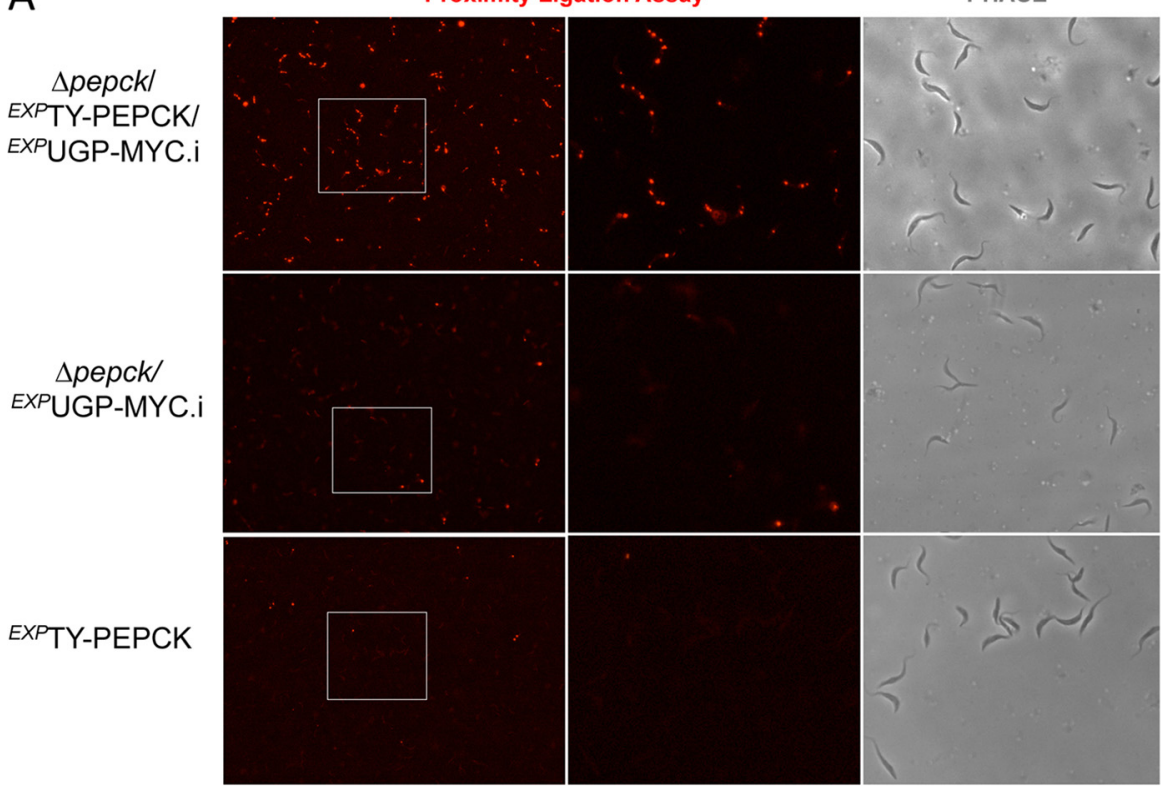

B

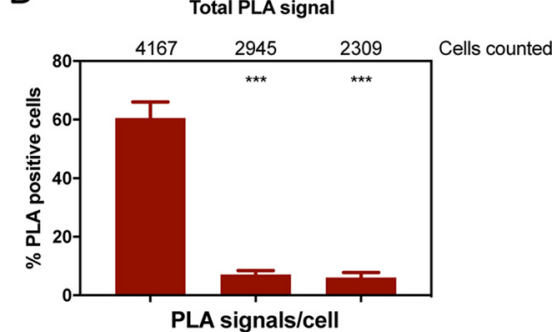

C

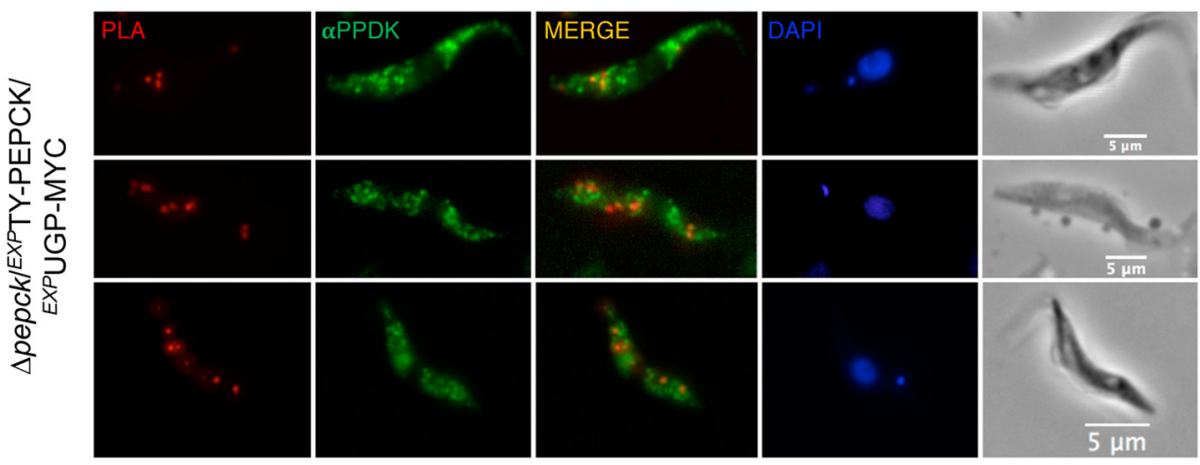

FIG 2 UGP interacts transiently with PEPCK. (A) In situ proximity ligation assay (PLA) analysis of the interaction between MYC-tagged UGP and TY-tagged

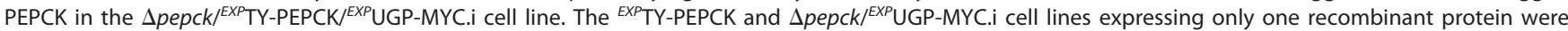
used as negative controls. The central (PLA) and right (phase contrast) panels are enlargements of the white rectangles shown in the left panel. (B) The percentage of PLA-positive cells is shown for each cell line, and the total cell number counted is indicated on the top of the graph. The percentages correspond to averages of 12 pictures randomly taken from 2 independent experiments. Significant differences between samples are indicated: *** $P<0.001$. The number of PLA signals per cell was analyzed by counting manually the number of dots using ImageJ for positive cells (lower panel). (C) The localization of the PLA signal was analyzed in detail and compared with that of the PPDK glycosomal marker ( $\alpha$ PPDK) by counterstaining after the Duolink in situ protocol.

Staining with an immune serum against the glycosomal PPDK showed that the PLA signals are found very close to the PPDK-containing organelles, without showing clear colocalization with them (Fig. 2C). This suggests the existence of different pools of glycosomes, as previously reported (33).

Determination of critical parts for PEPCK-UGP interaction. To investigate which part of UGP and PEPCK interacts with its piggybacking partner, truncated versions of each protein were expressed in the parental and $\Delta$ pepck cell lines, respectively. Since PEPCK forms homodimers (34), the truncated PEPCK proteins were expressed in the $\Delta$ pepck cell line to prevent heterodimer formation. UGP is reported to be monomeric $(7,22)$ and was detected only as monomer in native gel analyses (Fig. S2); therefore, the native and recombinant proteins will not directly interact. We expressed in the parental background the recombinant UGP with the $10 \times T Y$ tag either at the N-terminal or the C-terminal end of UGP (EXPTY-UGP ${ }_{1-485}$ and EXPUGP ${ }_{1-485}$-TY cell lines, respectively) by in situ replacement of one UGP allele. The subcellular distribution of UGP in these cell lines was determined by Western blotting of glycosomal and cytosolic fractions. The N-terminal tag affected the glycosomal import of UGP, since the glycosomal 

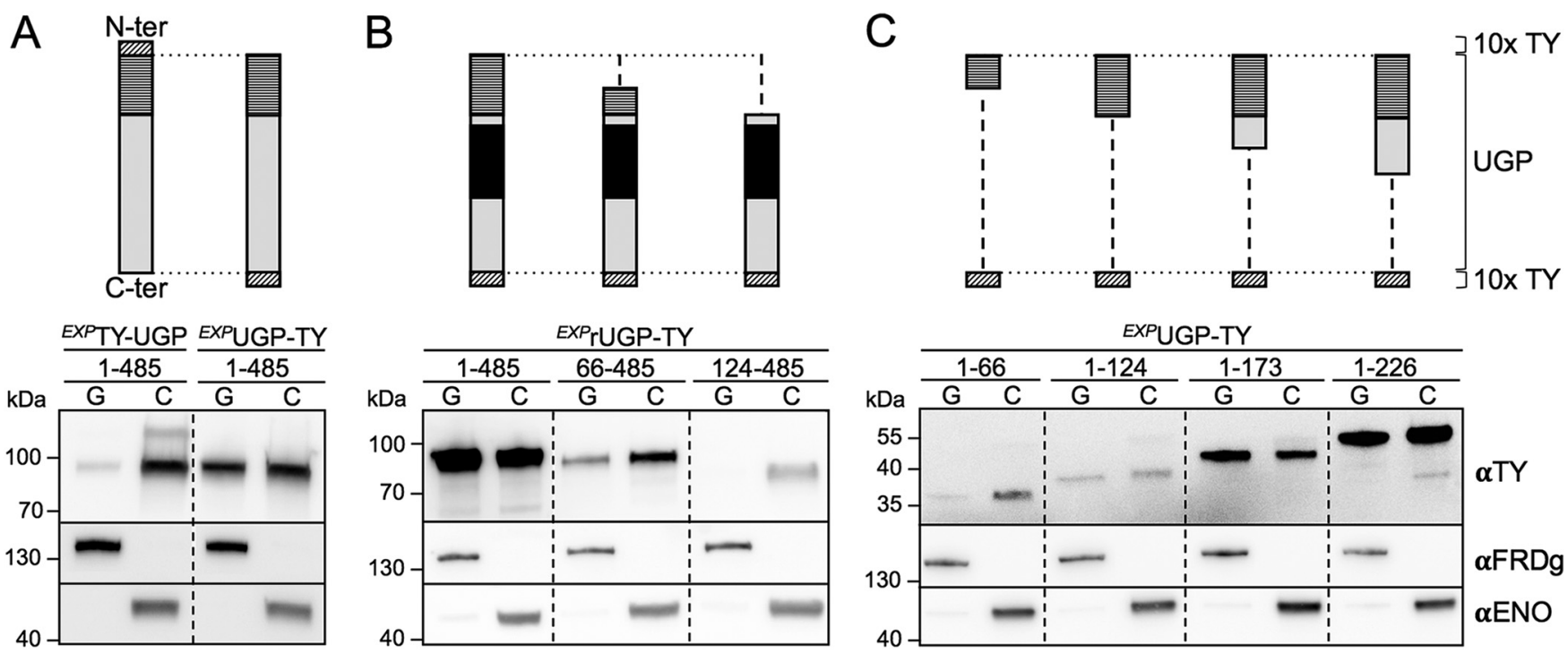

FIG 3 The $123 \mathrm{~N}$-terminal residues of UGP are required for import into the glycosomes. The top of the figure shows schematic representations of the $10 \times$ TY-tagged native or recoded UGP and the corresponding truncations. The 123-residue peptide required for UGP import, the recoded part of UGP, and the $10 \times$ TY tags are highlighted by horizontally striped boxes, black boxes, and obliquely hatched boxes, respectively. The lower part of the figure shows results of Western blot analyses of glycosomal (G) and cytosolic (C) fractions produced from cell lines expressing 10×TY-tagged recombinant UGP using the anti-TY antibody $(\alpha \mathrm{TY})$, as well as immune sera from glycosomal $(\alpha \mathrm{FRDg})$ and cytosolic $(\alpha \mathrm{ENO})$ markers. (A) Recombinant UGP proteins tagged at their $\mathrm{N}$-terminal ( $\mathrm{N}$-ter) (EXPTY-UGP ${ }_{1-485}$ ) or C-terminal (C-ter) (EXP UGP $\left.{ }_{1-485}-\mathrm{TY}\right)$ extremities; (B and C) truncated UGP tagged at its C-terminal extremity. The truncations designed from the $\mathrm{N}$ terminus lack the first 65 (66 to 485) or 123 (124 to 485) residues (B), while truncations designed from the $C$ terminus contain the first 66 ( 1 to 66 ), 124 ( 1 to 124), 173 (1 to 173), or 226 (1 to 226) N-terminal residues (C). A PCR analysis was performed to confirm the correct insertion of the UGP $\mathrm{Xxx}-485^{-T Y}$ fragments at the UGP locus (see Fig. S3 in the supplemental material).

localization of TY-UGP ${ }_{1-485}$ was decreased by $\sim 9$-fold compared to that of the native UGP in parental cells (Fig. 3A, left panel). However, no changes were observed in the glycosome/cytosol ratio for UGP ${ }_{1-485}-\mathrm{TY}$ (Fig. 3A, right panel, compared with Fig. 1A). $\mathrm{C}$-terminally tagged recombinant UGP versions truncated from their $\mathrm{N}$-terminal $\left(\mathrm{UGP}_{\mathrm{xxx}-485}-\mathrm{TY}\right)$ (Fig. 3B) or C-terminal (UGP $\left.{ }_{1-\mathrm{xxx}}-\mathrm{TY}\right)$ (Fig. 3C) extremities were inserted in situ to produce new cell lines. It is useful to note that the UGP coding sequence used for the UGP $\mathrm{XxX}_{\mathrm{x}-485}-\mathrm{TY}$ constructs was recoded from amino acid positions 165 to 337 to become resistant to the RNA interference (RNAi) construct (see below), which was useful to confirm the correct insertion of the recombinant fragment in the UGP locus (Fig. S3). The truncated recoded UGP protein with amino acids 124 to 485 $\left(\mathrm{rUGP}_{124-485}\right)$-TY was no longer imported into glycosomes (Fig. 3B), while glycosomal import of the UGP ${ }_{1-124}-\mathrm{TY}, \mathrm{UGP}_{1-173}-\mathrm{TY}$, and $\mathrm{UGP}_{1-226}-\mathrm{TY}$ proteins was not affected (Fig. $3 \mathrm{C}$ ), suggesting that the $\mathrm{N}$-terminal domain up to amino acid position 123 contains residues interacting with PEPCK. The truncated recombinant UGP missing $\left(\mathrm{rUGP}_{66-485}-\mathrm{TY}\right)$ or containing (UGP $\left.{ }_{1-66}-\mathrm{TY}\right)$ only the $66 \mathrm{~N}$-terminal residues were imported into glycosomes, although with a lower efficiency than occurred with the parental cell line, suggesting that key residues of the PEPCK binding site are located on either side of position 66 (Fig. 3B and C). The presence of the PEPCK binding site in the $\mathrm{N}$-terminal extremity of UGP may explain the low glycosomal import of the recombinant TY-UGP ${ }_{1-485}$ protein (Fig. 3A).

We performed a similar analysis to determine the PEPCK region involved in UGP glycosomal import by expressing truncated versions of recombinant PEPCK using the pLew100 vector. PEPCK was truncated from its N-terminal extremity in order to maintain Cterminal PTS1, required for glycosomal import of both PEPCK and UGP. Unfortunately, none of the truncated PEPCK peptides were detectable by Western blotting in total cell extracts, probably due to protein instability. To resolve this stability issue, the truncated PEPCK peptides were fused to the C-terminal extremity of the enhanced green fluorescent protein (eGFP) and used to produce four different cell lines (Fig. 4A). We determined the glycosomal import of UGP in these $\Delta$ pepck/EXP GFFP-PEPCK $_{x x x-525}$ cell lines by Western blotting of 


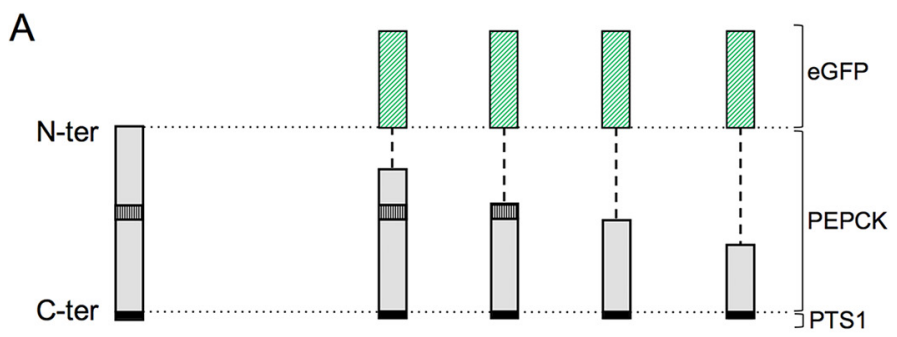

B

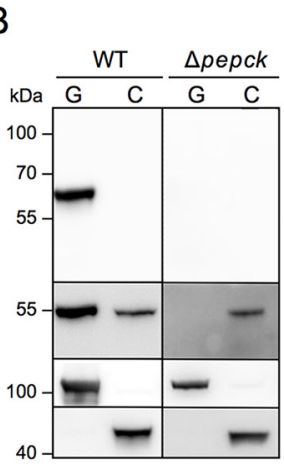

C $\triangle$ Ppepck/EXPeGFP-PEPCK
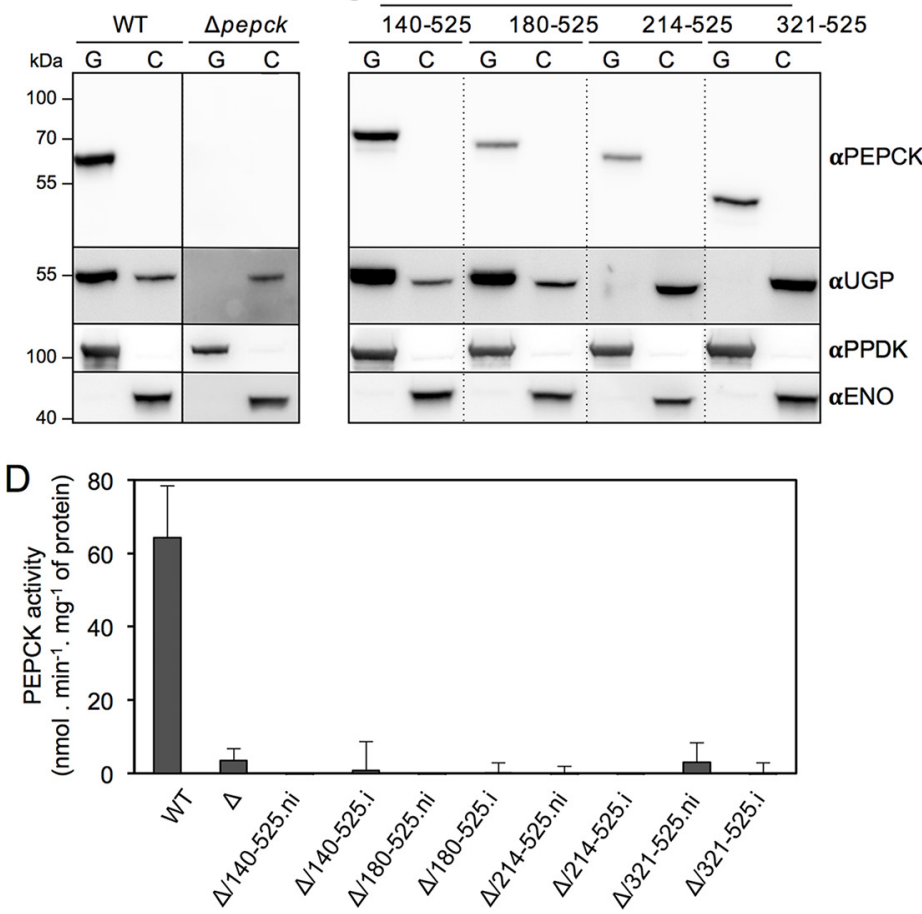

FIG 4 A 34-residue peptide of PEPCK is required for glycosomal import of UGP. (A) Schematic representations of the endogenous PEPCK and eGFP-PEPCK recombinant proteins expressed in the $\Delta$ pepck background, in which the 34-residue peptide required for UGP import (vertically striped boxes) is highlighted. (B and C) Western blot analyses of glycosomal (G) and cytosolic (C) fractions obtained from the parental and $\Delta$ pepck cell lines (B), as well as from cell lines expressing truncated recombinant eGFP-PEPCK proteins ( $\Delta$ pepck/ ${ }^{\text {EXP }}$ eGFP-PEPCK ${ }_{\mathrm{xxX}-525}$ ) with anti-PEPCK and anti-UGP immune sera. Glycosomal ( $\alpha$ PPDK) and cytosolic $(\alpha$ ENO) markers are used to check the quality of glycosomal and cytosolic fractions. (D) PEPCK activity determined in total extracts of the WT, the $\Delta$ pepck $(\Delta)$, and the noninduced (.ni) and induced (.i) $\Delta$ pepck/EXP eGFP-PEPCK ${ }_{\mathrm{xxX}-525}$ cell lines.

glycosomal and cytosolic fractions. As mentioned above, UGP was no longer detected in glycosomes isolated from the parental $\Delta$ pepck mutants (Fig. 4B). The glycosomal import of UGP was not affected in the absence of the first 140 and $180 \mathrm{~N}$-terminal residues of PEPCK ( $\Delta$ pepck/EXP $^{\text {EXFP-PEPCK }}{ }_{140-525}$ and $\Delta$ pepck/EXP $^{\text {EGFP-PEPCK }}{ }_{180-525}$ cell lines), while deletion of the first 214 and $321 \mathrm{~N}$-terminal residues abolished the glycosomal import of UGP, which remained exclusively in the cytosolic fractions (Fig. 4C). This suggested that the 34-residue peptide between amino acids positions 180 and 214 of PEPCK is required for UGP import into glycosomes. Importantly, none of the eGFP-PEPCK truncations have PEPCK activity, indicating that the import of UGP is not related to PEPCK activity inside the glycosomes (Fig. 4D).

Targeting a recombinant UGP exclusively to the glycosomes. To elucidate in which subcellular compartment the UDP-GIc/UDP-Gal biosynthetic pathway is active (glycosomes and/or cytosol), it was necessary to express UGP exclusively in the cytosol or in the glycosomes of the parasite. The exclusive cytosolic localization of UGP in the viable $\Delta$ pepck mutant demonstrated that UGP is functionally active in the cytosol. To 
assess the role of UGP in glycosomes, we optimized the glycosomal import of UGP with the objective that all of the recombinant UGP is localized within the glycosomes. To do so, a recombinant UGP gene recoded to become resistant to the RNAi construct $(r U G P)$ was fused at its $3^{\prime}$ extremity with a $3 \times$ MYC tag followed by different glycosomal targeting peptides (PTS1), namely, the last 12 C-terminal residues of glycosomal FRDg (rUGP-FRDgPTS1), the full-length PTS1-containing glycosomal glycerol-3-phosphate dehydrogenase (GPDH) gene ( $r U G P-G P D H)$, and the full-length PTS1-containing glycosomal phosphoglycerate $(P G K C)$ gene $\left(r U G P-P G K c^{*}\right)$. Since glycosomal expression of PGK is lethal for the PCF trypanosomes (35), the codon of the lysine residue (K215) essential for the PGK enzymatic activity (36) was replaced by the alanine codon. These recombinant proteins were conditionally expressed in the parental cell line, and their distribution between the glycosomal and cytosolic compartments was determined by digitonin titration (Fig. 5A). The rUGP-FRDgPTS1 and cytosolic enolase proteins showed the same cytosolic profiles, which implies that the extended FRDg PST1 motif is not sufficient for glycosomal import of UGP. In contrast, the rUGP-PGKc* recombinant protein is mostly associated with the glycosomes, but a minor part remained in the cytosol. Finally, both the rUGP-GPDH $(\sim 100-\mathrm{kDa})$ and the glycosomal FRDg proteins were released with a minimum of $0.16 \mathrm{mg}$ digitonin per $\mathrm{mg}$ protein, which is consistent with the exclusively glycosomal localization of this recombinant protein. The UGP activity was increased by 5-fold in the EXPrUGP-GPDH.i cell line compared to those in the noninduced (.ni) and parental cell lines, which validated the functionality of the rUGP-GPDH protein (Fig. 5B). Expression of rUGP-GPDH had no effect on the morphology, growth, or survival of the ${ }^{E X P}$ rUGP-GPDH cell line.

The UGP protein is essential for $\boldsymbol{T}$. brucei. The stem-loop RNAi strategy was used with the conditional pLew100 vector to address the role of UGP in the procyclic trypanosomes. Two ${ }^{R N A}$ UGP cell lines obtained from individual transfections (H10 and E4) showed a strong reduction of growth 7 days after tetracycline induction, indicating that UGP is essential for PCF viability (Fig. 6A, top panel). For both RNAi cell lines, the growth rate of the parental strain was restored 18 days postinduction, concomitantly with the reexpression of the native UGP (Fig. 6A, lower panel). This reexpression of RNAi-targeted genes is often observed for trypanosome essential genes (30). It is noteworthy that the UGP expression was barely detectable in the noninduced RNAIUGP-H10 total cell extracts. Western blot analyses of enriched glycosomal fractions, which proved to be more sensitive than on total cell extracts, showed that UGP expression was reduced by $\sim 30$-fold compared to that in the parental cells, without any significant effect on growth (Fig. 6B, left panel). This suggests that UGP activity is present in large excess in parental PCFs. The distribution of UGP between glycosomal and cytosolic compartments is not affected by this $\sim 30$-fold reduction (Fig. 6B). After 5 days of induction, UGP was no longer detectable in the glycosomal fractions and was reduced by $\sim 2$-fold in the cytosol (Fig. 6B). These small amounts of UGP were not sufficient to sustain the growth of PCFs.

To determine whether UGP is also required for the growth of the procyclic trypanosomes under the insect-like glucose-free conditions, the parasites were grown in the absence of glucose, as described before (37). The growth of the RNAiUGP.i and $\Delta p e p c k /{ }^{R N A} U G P . i$ cell lines is similar regardless of the amounts of glucose in the medium (Fig. S4), indicating that the UGP is probably also essential in the insect vector, which is considered to be free of glucose (38). In addition, the subcellular distribution of UGP in the parental cells is not affected by the absence of glucose (Fig. S5).

Expression of glycosomal rUGP-GPDH rescues the lethality of the RNAiUGP mutant. The EXPrUGP-GPDH construct (pHD1336-rUGP-GPDH), which produces an exclusively glycosomal rUGP, was introduced into the ${ }^{R N A i U G P-H 10}$ cell line. Western blot analyses showed that native UGP was no longer detectable in the glycosomal and cytosolic fractions of the ${ }^{R N A}$ UGG/EXPrUGP-GPDH.i cell line, while the dying RNAiUGP.i cells still expressed residual amounts of UGP in the cytosol (Fig. 6B, right panel). The exclusive glycosomal subcellular localization of the recombinant rUGP-GPDH protein in the ${ }^{R N A}$ UGP/EXP ${ }^{E U G P-G P D H}$.i cell line observed by cellular fractionation (Fig. 6B) was 
A

mg digitonin . $\mathrm{mg}^{-1}$ of protein
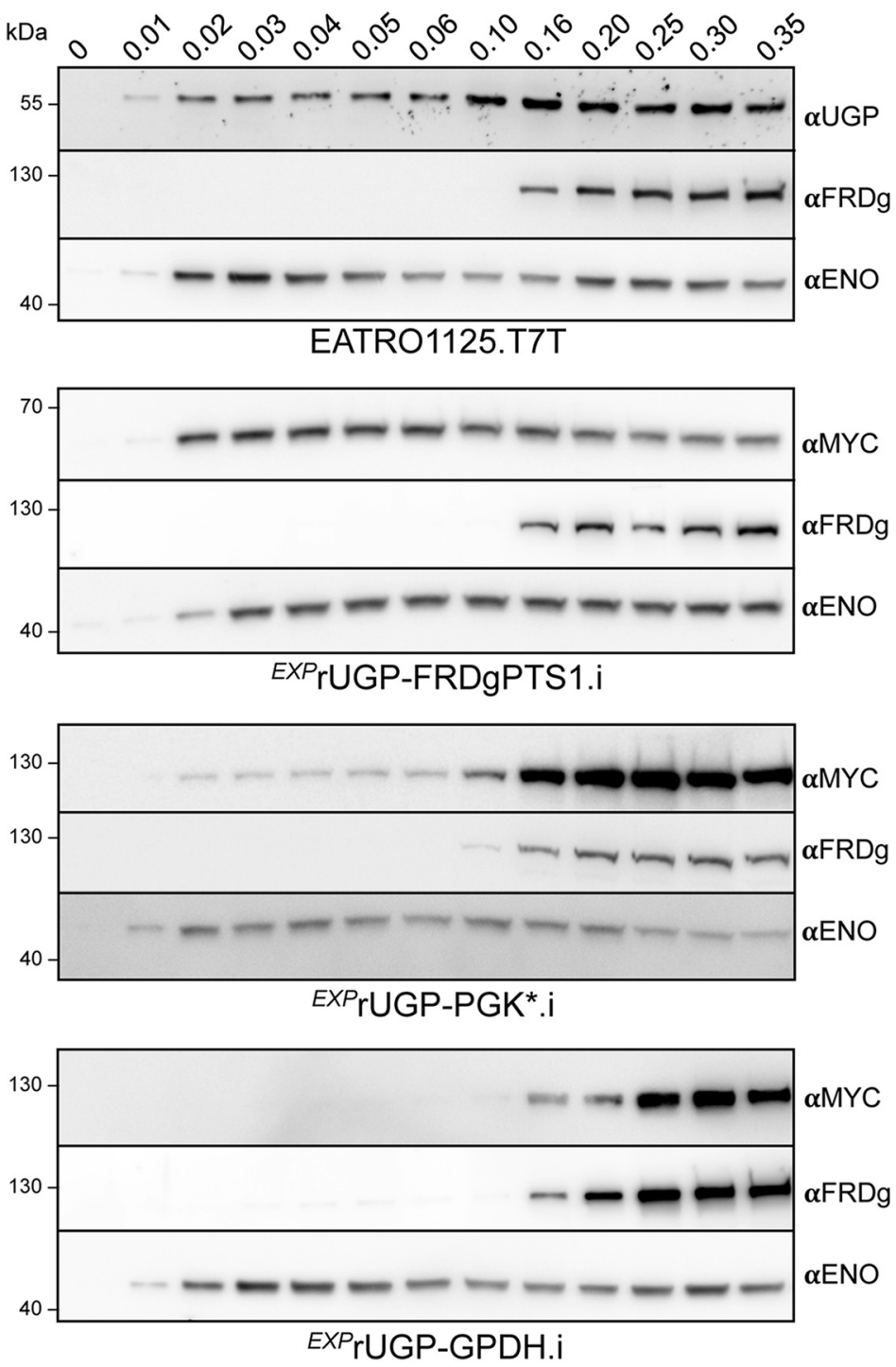

B

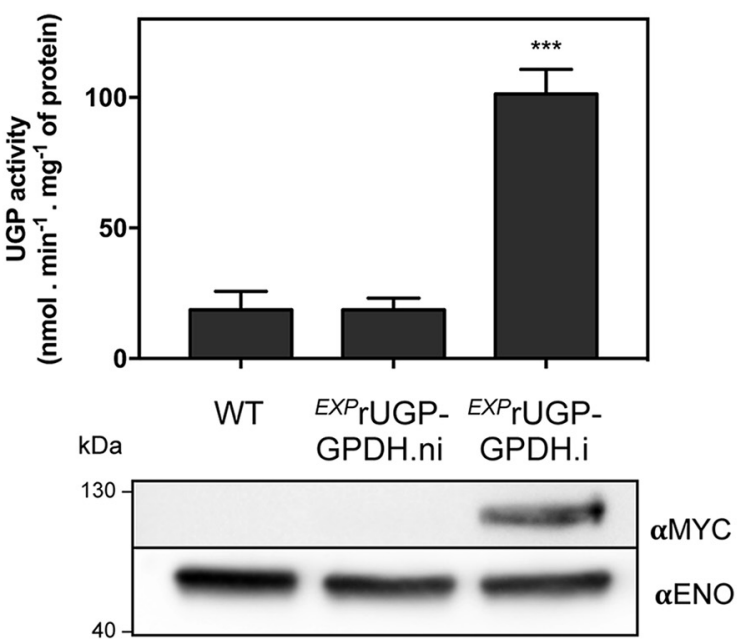

FIG 5 Expression of a glycosomal recombinant UGP. (A) The subcellular localization of recombinant UGP was monitored by Western blotting of supernatants obtained after digitonin titration of the

(Continued on next page) 
A

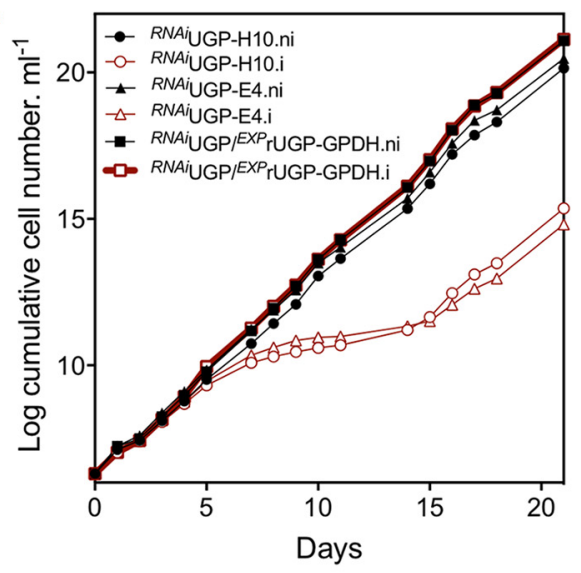

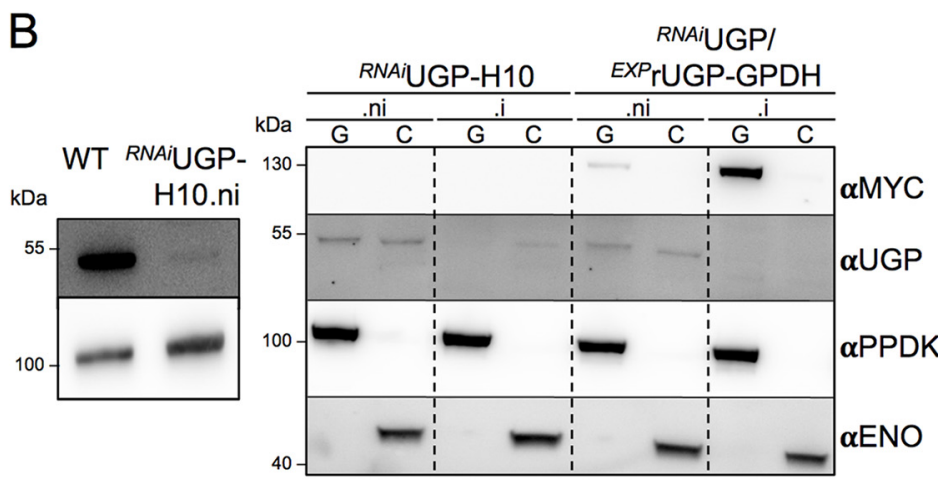

C mg digitonin. $\mathrm{mg}^{-1}$ of protein

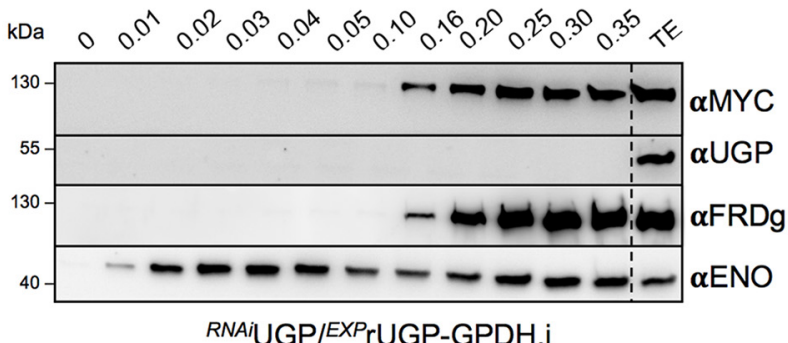

FIG 6 Production and functional analyses of ${ }^{R N A i}$ UGP cell lines. (A) Growth curve of the tetracycline-induced and noninduced ${ }^{R N A i}$ UGP-H10, ${ }^{R N A i}$ UGP-E4, and ${ }^{R N A}{ }^{-}$UGP/EXP rUGP-GPDH cell lines. The expression of native UGP and recombinant rUGP-GPDH upon induction was monitored by Western blotting using antiUGP and anti-MYC immune sera, respectively, and anti-PPDK as a loading control (bottom). (B) Western blot analyses of glycosomal (G) and cytosolic (C) fractions produced from the parental cells (WT), as well as the tetracycline-induced and noninduced ${ }^{R N A i U G P-H 10}$ and ${ }^{R N A}{ }^{2} U G P /{ }^{E X P}$ rUGP-GPDH cell lines, using immune sera described in the preceding figures. (Left) Quantification of the relative expression of UGP in glycosomes of the noninduced ${ }^{R N A i}$ UGP-H10 mutant. (C) The glycosomal localization of recombinant rUGP-GPDH ( $\alpha \mathrm{MYC}$ ) was confirmed by Western blotting analyses of supernatants obtained after digitonin titration of the ${ }^{R N A i} U G P /{ }^{E X P}$ rUGP-GPDH.i cell line, using the immune sera described in precedent figures. The control lane TE corresponds to total extract from the ${ }^{E X P}$ rUGP-GPDH.i cell line.

confirmed by digitonin titration (Fig. 6C). In the context of the absence of cytosolic UGP, the viability of the ${ }^{R N A}$ U UGP/EXP rUGP-GPDH.i cell line (Fig. 6A) strongly supported the hypothesis that the glycosomal pathway is functional. However, it be cannot excluded that residual expression of UGP in the cytosol is responsible for the growth of the RNAiUGP/EXPrUGP-GPDH.i cell line.

The $\Delta u g p /{ }^{E X P}$ rUGP-GPDH cell line is viable. To confirm the functionality of the glycosomal pathway, rUGP-GPDH was expressed in the null UGP background ( $\Delta u g p)$. Considering that UGP is an essential protein, knockout mutants were produced in two cell lines expressing tetracycline-inducible recombinant UGP, i.e., glycosomal/cytosolic rUGP and glycosomal rUGP-GPDH. The UGP alleles were replaced by the PAC and BLE markers after transfection with the recombinant plasmids expressing rUGP ( $\Delta$ ugp/ ${ }^{E X P}$ rUGP) or rUGP-GPDH ( $\Delta$ ugp/EXPrUGP-GPDH), in the presence of tetracycline to express the recombinant rUGP or rUGP-GPDH, respectively. Deletion of both UGP alleles was confirmed by PCR (Fig. 7A) and Western blotting (Fig. 7B). Tetracycline removal did not induce the death of the parasites (Fig. 7C), since the recombinant rUGP and rUGP-GPDH proteins were still expressed after 18 days in the absence of tetracycline (Fig. 7B, inset, and Fig. 7C). However, the growth of the $\Delta$ ugp/ ${ }^{\text {EXP }}$ rUGP-GPDH.ni cell line was slightly affected after tetracycline removal, which is consistent with the

FIG 5 Legend (Continued)

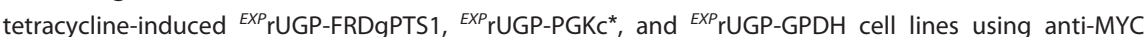
antibody. (Top) The anti-UGP immune serum was used to detect UGP in parental cells fractions. AntiFRDg and anti-ENO immune sera were used as glycosomal and cytosolic markers, respectively. (B) UGP activity measured in total cell extracts of parental (WT) and tetracycline-induced and noninduced EXPrUGP-GPDH cell lines ( $n=3$; standard errors of the means [SEM]). Significant differences between samples are indicated. ${ }^{* * *}, P<0.001$. (Bottom) A Western blot analysis of EXPrUGP-GPDH expression with anti-MYC and anti-ENO (loading control) immune sera is shown below the graph. 

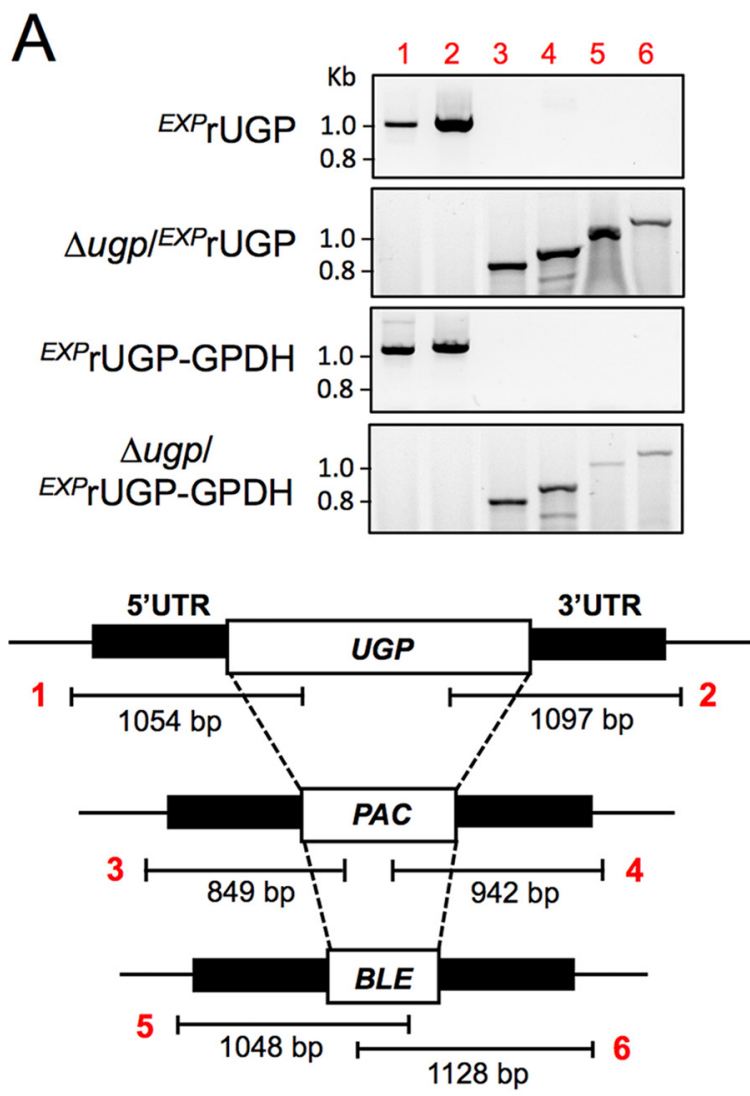
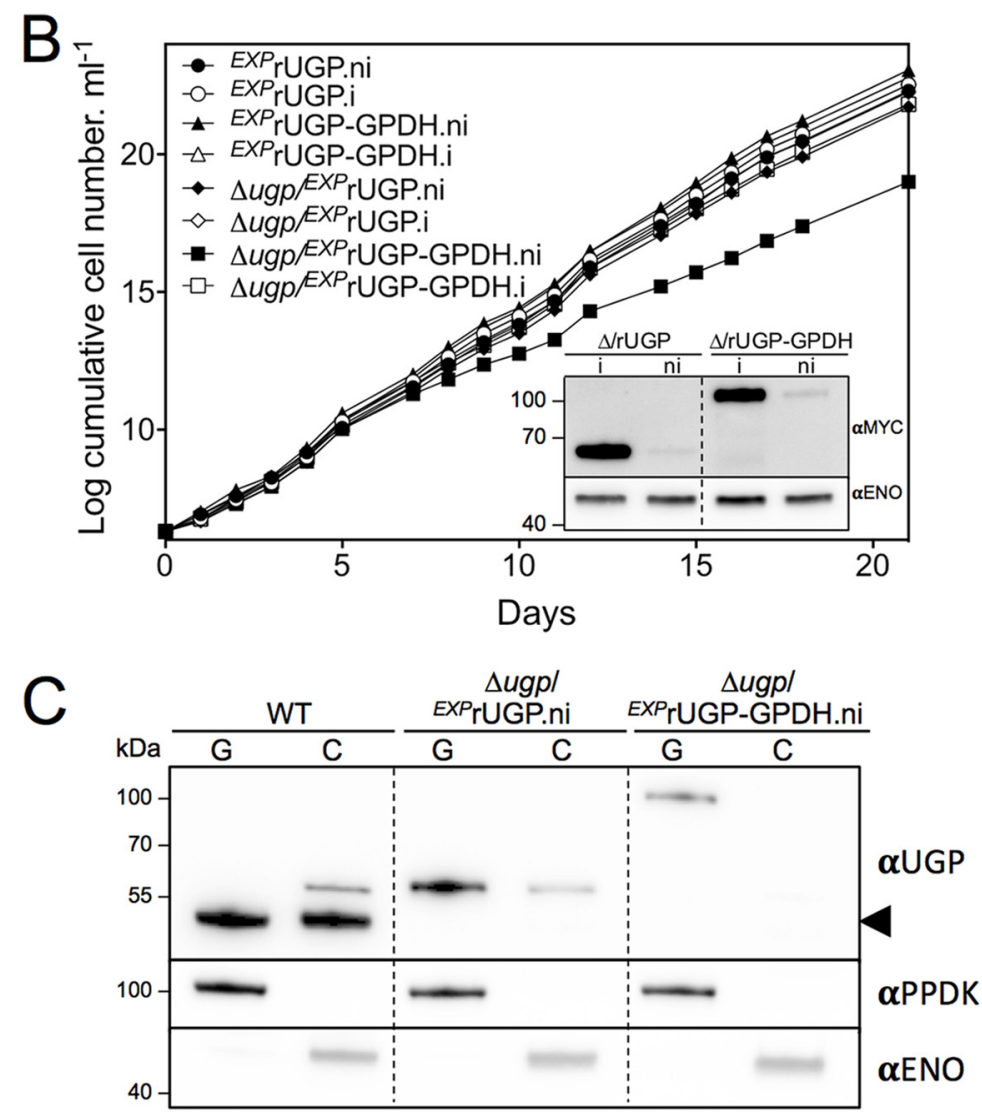

$\mathrm{mg}$ digitonin . $\mathrm{mg}^{-1}$ of protein
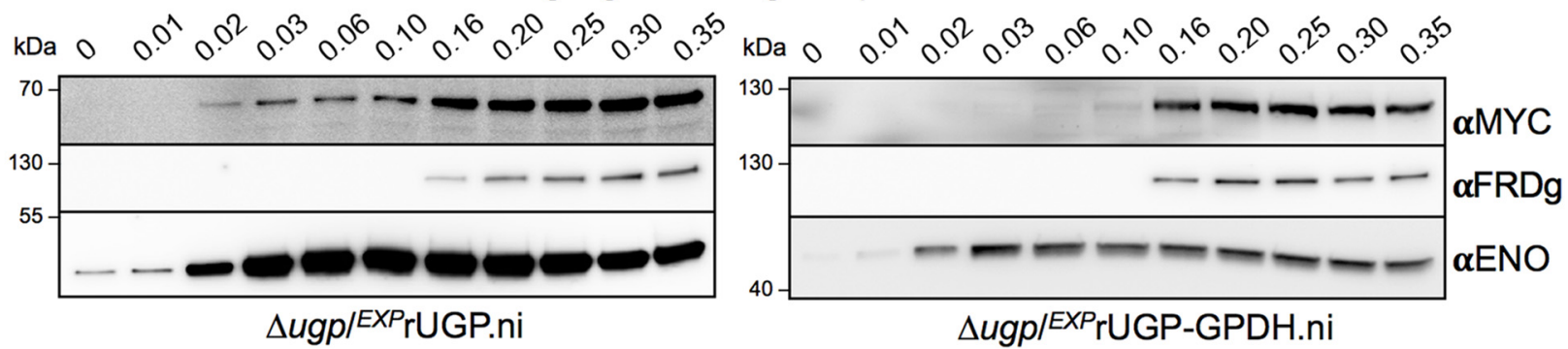

FIG 7 Production and functional analyses of $\Delta$ ugp cell lines. (A) PCR analysis of genomic DNA isolated from the parental ( ${ }^{E X P}$ rUGP and EXPrUGP-GPDH) and null mutant $\left(\Delta u g p /{ }^{E X P}\right.$ rUGP and $\Delta u g p /{ }^{E X P}$ rUGP-GPDH) cell lines. Both knockout cell lines were obtained in the presence of tetracycline. Primers are designed on the basis of sequences flanking the $5^{\prime}$ UTR and $3^{\prime}$ UTR fragments used to target UGP gene depletion (black boxes) and on the open reading frame (ORF) of the UGP gene, as well as the puromycin (PAC) and phleomycin (BLE) resistance genes (white boxes). $\triangle$ ugp/EXPrUGP represents a control cell line expressing a recombinant UGP with a dual cytosolic and glycosomal localization. (B) Glycosomal (G) and cytosolic (C) fractions obtained after subcellular fractionation of the UGP null cell lines in the absence of tetracycline (5 days). The arrowhead highlights the native UGP only in parental (WT) cells. (C) The growth of the cell lines was followed during 21 days in the presence (.i) or the absence (.ni) of tetracycline. Western blot analyses with anti-MYC and antiENO (loading control) of the tetracycline-induced and noninduced (18 days after tetracycline removal) $\Delta$ ugp/EXPrUGP and $\triangle$ ugp/EXPrUGP-GPDH mutants are shown in the inset. (D) The UGP null cell lines were analyzed by digitonin titration 5 days after removal of tetracycline. A Western blot of supernatants

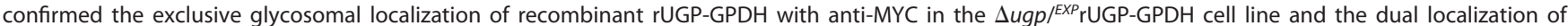
rUGP in the $\Delta u g P /{ }^{E X P}$ rUGP cell line.

essential role of UGP. The absence of growth retardation for the $\Delta$ ugp/EXPrUGP.ni cell line, while the amounts of residual recombinant UGP were equivalent in the two cell lines (see Fig. 7C), might be interpreted as the cytosolic pathway having a higher efficiency than

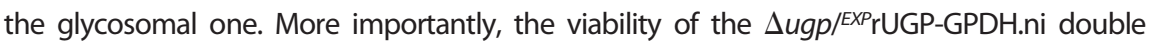
mutant supports our hypothesis that the glycosomal pathway is functional.

These data, in agreement with the functional role of the glycosomal UGP, had to be confirmed by determining the subcellular localization of rUGP-GPDH in the $\triangle$ ugp/EXPrUGPGPDH.ni cell line. After 5 days in the absence of tetracycline, the viable $\triangle$ ugp/EXPrUGP-GPDH. 
ni cell line expressed the recombinant rUGP-GPDH exclusively in the glycosomes (Fig. 7B to $D$, right panels). These data confirmed that the UDP-GIc/UDP-Gal biosynthetic pathway, which includes UGP, is active in the glycosomes. As expected, the MYC-tag rUGP showed dual glycosomal and cytosolic localizations in the $\Delta u g p /{ }^{E X P}$ rUGP.ni cell line (Fig. 7B to D).

The glycosomal and cytosolic UGP-containing pathways are functional. To confirm the functionality of the glycosomal and cytosolic pathways involving UGP, cell lines expressing the native and/or recombinant UGP (i) in both subcellular compartments (wild type [WT], ${ }^{E X P}$ rUGP.i, and ${ }^{E X P}$ rUGP-GPDH.i), only in the cytosol ( $\Delta$ pepck), (ii) only in the glycosomes (RNAiUGP/EXPrUGP-GPDH.i), or (iii) not at all (RNAiUGP-H10.i) were further analyzed (Fig. 8A to C). This included determining the expression levels of UGP in the glycosomal and cytosolic fractions by Western blotting and determination of enzymatic activities, as well as by quantifying intracellular metabolites, including the substrate (G1P) and the product (UDP-Glc) of the UGP enzymatic reaction, by a mass spectrometry-based metabolomics profiling approach (ion chromatography-high-resolution mass spectrometry [ICHRMS]).

The specific activity of UGP (the ratio between the enzymatic activity and the relative amount of proteins detected by Western blotting) in the cytosolic fractions of EXPrUGP.i is $\sim 3.5$-times lower than in the parental WT cells, suggesting that the C-terminal MYC tag affects UGP activity (Fig. 8C). Similarly, the native UGP shows a specific activity in the glycosomal fraction 4 times lower than in the cytosolic fraction of the parental cells, which suggests that the glycosomal sequestration of UGP affects its activity by a yet-unknown mechanism. These data provide a rational explanation for the growth retardation observed for the $\Delta$ ugp/EXPrUGP-GPDH.ni cell line, while the growth of the $\Delta u g p /{ }^{E X P}$ rUGP.ni cell line was not affected, although the amounts of residual recombinant UGP were equivalent in the two cell lines (Fig. 7C). We also confirmed that the coupling enzyme (UDP-Glc dehydrogenase) used in the UGP activity assays was not affected by the presence of the same amounts of the glycosomal or cytosolic samples ( 273 versus $245 \mathrm{mU} \cdot \mathrm{mg}^{-1}$ of protein, respectively). The activity of the recombinant rUGPs, which is $\sim 30$ times more expressed in the EXPrUGP.i line than the native UGP, was not affected in glycosomes, as the enzyme specific activities were similar in the glycosomal and the cytosolic fractions (Fig. 8C). It is also noteworthy that the UGP activity was detected in the cytosol of the ${ }^{\text {RNAiUGP/EXP }}$ rUGP-GPDH.i line, while the native UGP was not detectable by Western blotting (Fig. 8B) and the recombinant rUGP-GPDH was exclusively glycosomal (Fig. 6C). This may be due to the rupture of a few glycosomes during the grinding step designed to disrupt primarily the plasma membrane.

To confirm the role of UGP subcellular localization in UDP-Glc production, we used mass spectrometry-based metabolomics to determine the intracellular amounts of G6P, G1P, and UDP-Glc (Fig. 8D), as well as other metabolites as controls (Fig. S6), in the cell lines mentioned above cultivated in SDM79 medium. This metabolomics approach was validated with the analysis of the $\Delta$ pepck cell line, in which the metabolic flux through the Gly3P/DHAP shuttle, used to maintain the glycosomal redox balance, has been reported to be increased in the absence of PEPCK (30). Indeed, the level of Gly3P is increased by $\sim 3$ times in the $\Delta$ pepck mutant compared to levels in all the other cell lines analyzed (Fig. S6). Regarding the sugar nucleotide biosynthetic pathways, only UDP-Glc and UDP-GIcNAc were identified and quantified with this methodology (Fig. S6), and the levels of UDP-Glc detected (80 to $170 \mu \mathrm{M}$ ) were comparable to those previously reported for procyclic trypanosomes (110 to $540 \mu \mathrm{M}$ ) (39) (Fig. 8D). UDP-Glc was no longer detectable in the RNAiUGP.i cell line (Fig. 8D), which shows that UGP was the only enzyme producing UDP-Glc in PCF trypanosomes. It is also of note that UDP-Glc was detected in noninduced RNAiUGP cells at levels similar to those in parental cells, despite the $\sim 30$-fold reduction of UGP protein levels (Fig. $6 \mathrm{~B}$ ), which shows that PCF trypanosomes express a large excess of UGP. Most importantly, UDP-Glc was produced in cells expressing UGP exclusively in the cytosol ( $\Delta$ pepck cells) or in 
A

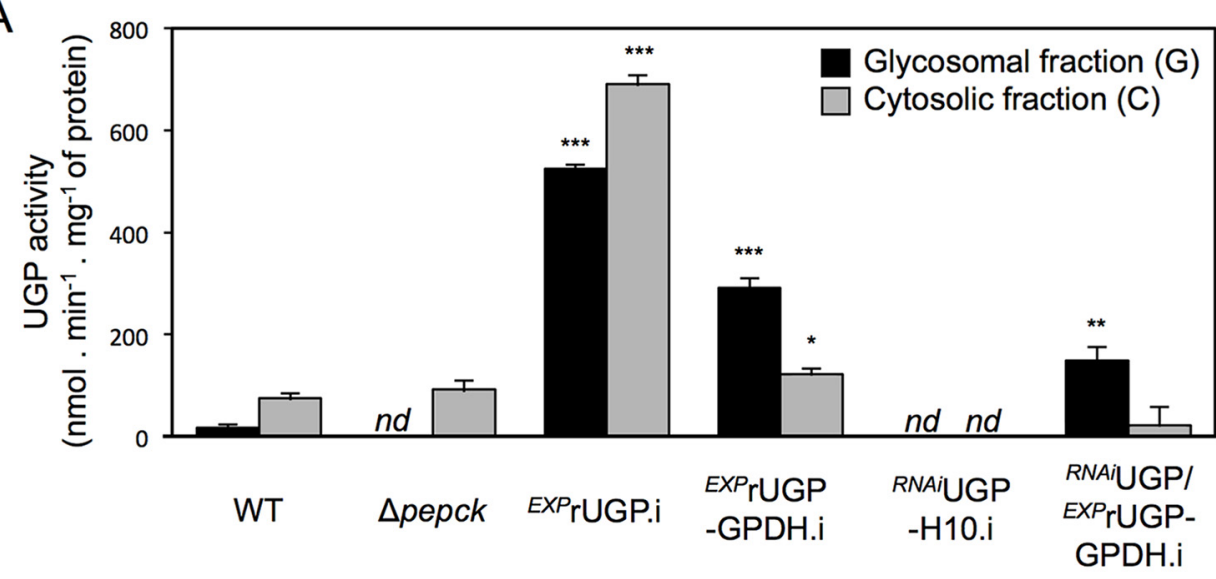

B
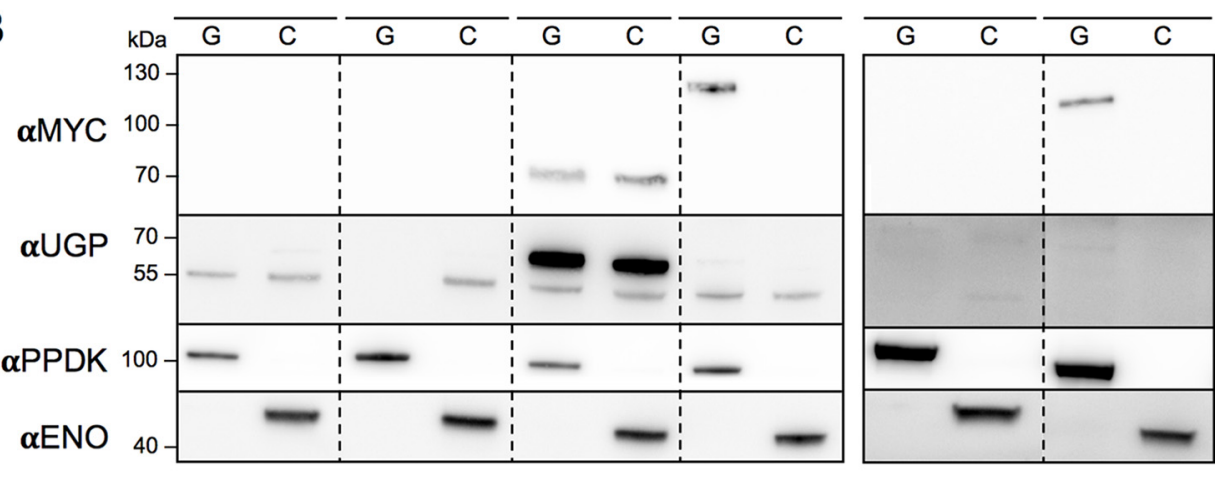

C

\begin{tabular}{|c|c|c|cc|cc|cc|cc|cc|}
\hline Protein level & 1 & 1 & $n d$ & 2.1 & 30.0 & 31.8 & 45.5 & 0.9 & $n d$ & $n d$ & 31.3 & 0.8 \\
Activity & 1 & 4 & $n d$ & 5.6 & 29.0 & 36.0 & 16.2 & 6.4 & $n d$ & $n d$ & 8.3 & 1.2 \\
\hline
\end{tabular}

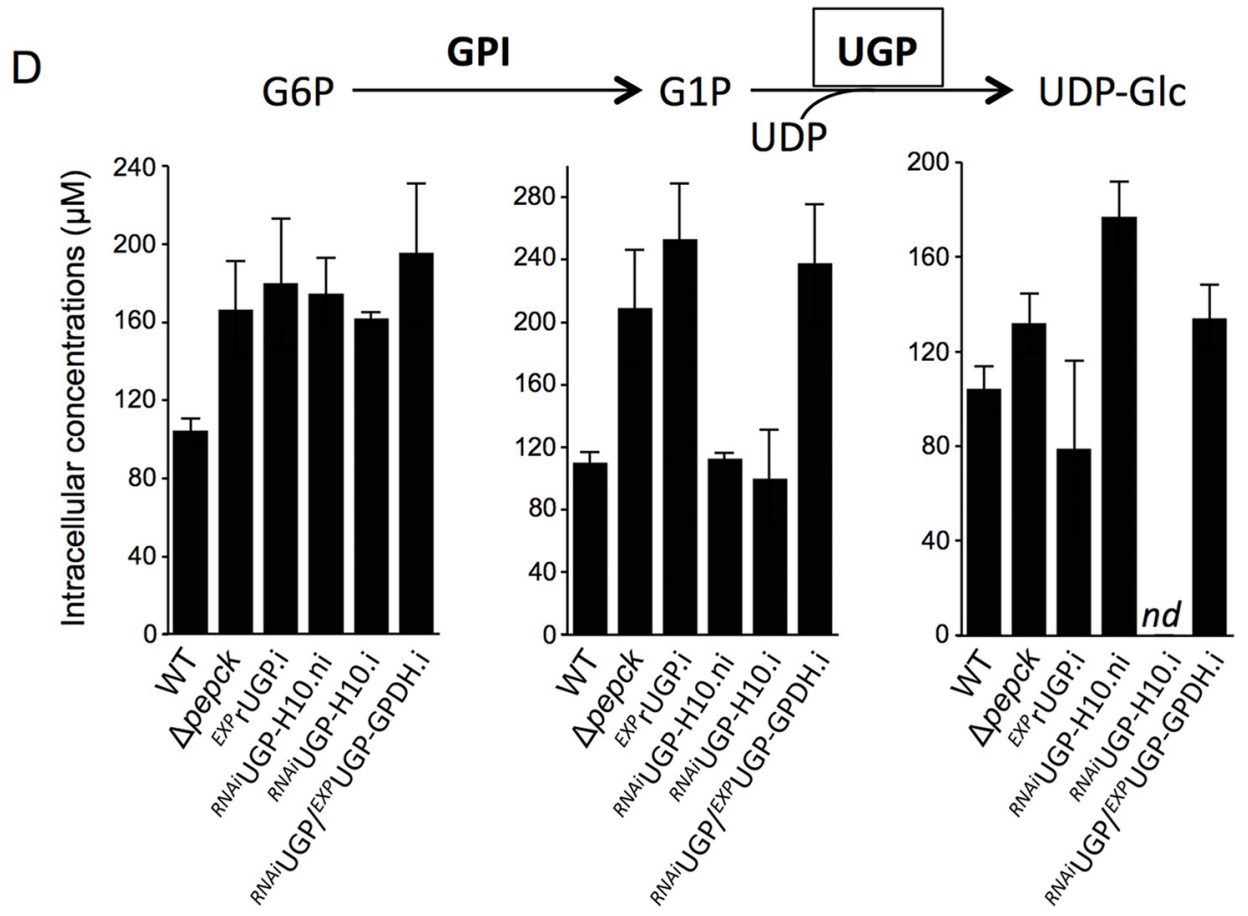

FIG 8 PCF produces UDP-Glc in glycosomes and the cytosol. (A) UGP activity was determined in enriched glycosomal and cytosolic fractions of the WT, $\Delta$ pepck, ${ }^{E X P}$ rUGP.i, ${ }^{E X P}$ rUGP-GPDH.i, ${ }^{R N A i U G P-H 10 . i, ~ a n d ~}$ 
glycosomes ( ${ }^{R N A i U G P / E X P}$ rUGP-GPDH.i cells) at levels similar to those of WT cells, which confirms the functionality of the pathway in both subcellular compartments.

\section{DISCUSSION}

Trypanosomatids are known to sequester a cascade of consecutive glycolytic enzymes into glycosomes, in addition to enzymes of other pathways, including those for gluconeogenesis, pentose phosphate, and sugar nucleotide biosynthesis $(4,40)$. In this study, we address three questions related to the glycosome biology by analyzing UGP, a key enzyme of sugar nucleotide biosynthesis involved in UDP-Glc synthesis. (i) The physiological role of this glycosomal pathway remains unknown since it is also present in the cytosol, the subcellular compartment where the biosynthesis of sugar nucleotides takes place in the other eukaryotes. (ii) The molecular mechanisms leading to the import of glycosomal enzymes lacking peroxisomal targeting signals (PTS1 or PTS2) have not yet been investigated in trypanosomatids. (iii) Mammalian peroxisomes multiply by the ER de novo route or by growth and division followed by protein import into newly produced organelles, but what about glycosomes? Here, we show that (i) the glycosomal pathway leading to the production of UDP-Glc and UDP-Gal is functional and is essential in PCF trypanosomes in the absence of the cytosolic pathway, (ii) UGP is imported into glycosomes by piggybacking on the PTS1-containing PEPCK, and (iii) PEPCK and UGP interact in only a few glycosomes, which may represent newly produced glycosomes competent for protein import.

What is the role of sugar nucleotide biosynthesis in glycosomes? The functionality of the glycosomal and cytosolic UGP-containing pathways was validated by the viability of mutants expressing UGP exclusively in glycosomes ( ${ }^{R N A} U$ UGP/EXP rUGP-GPDH.i cells) or the cytosol ( $\Delta$ pepck cells) and the detection of UDP-Glc in both cell lines. This first direct evidence of a functional production of sugar nucleotides inside glycosomes raises two questions. First, how do de novo-synthesized UDP-Glc and UDP-Gal leave the glycosomes to reach the ER and Golgi apparatus, where they are required for protein glycosylation? The glycosomal membrane is considered to be impermeable to bulky metabolites, such as nucleotides, since the size limitation of the general peroxisomal diffusion pore is on the order of $400 \mathrm{Da}(40,41)$. Consequently, exchange of sugar nucleotides between the glycosomal and cytosolic compartments requires transporters. However, the only transporters known to be associated with the glycosomal membrane are the ABC transporters GAT1, GAT2, and GAT3, with GAT1 likely transporting acyl coenzyme A's (acyl-CoAs) $(42,43)$, and proteomics analyses of glycosomal membrane fractions did not reveal additional candidates (44). Further work is certainly required to confirm the presence of such sugar nucleotide transporters in the glycosomal membrane. Second, what is the role of sugar nucleotide biosynthesis inside the glycosomes, since the cytosolic pathway is functional in the procyclic trypanosomes, as observed in all eukaryotes? UGP has also been localized in the Golgi apparatus, chloroplasts, and membrane fractions, as well as in cell walls, where it also provides UDP-Glc to produce glycoconjugates in plants and yeasts (45). Interestingly, the yeast UGP also shows a dual subcellular localization depending on phosphorylation at the N-terminal S11 residue, with the nonphosphorylated cytosolic and phosphorylated cell wall

FIG 8 Legend (Continued)

${ }^{R N A}{ }^{\text {UUGPFEXP }}$ rUGP-GPDH.i cell lines ( $n=3$, SEM). The cytosolic and glycosomal UGP activities were normalized to the cytosolic malic enzyme and glycosomal glycerol kinase activities, respectively. This normalization consists of recalculating the UGP activities by considering that the malic enzyme and glycerol kinase activities are the same in the different cell lines. Significant differences between the WT and mutants are indicated for each compartment. ${ }^{* * *}, P<0.001 ;{ }^{* *}, P<0.01 ;{ }^{*}, P<0.05$. (B) Representative Western blots of the corresponding cell lines. Recombinant rUGP-GPDH was detected with anti-MYC antibody. Glycosomal ( $\alpha$ PPDK) and cytosolic $(\alpha \mathrm{ENO})$ markers are also shown. (C) Relative amounts of UGP (determined by Western blotting) and specific activity (average of 3 experiments). For these comparative analyses, the protein and activity levels detected in the glycosomal fraction of WT cells were used as references and given the arbitrary value of 1 (boxed values). (D) IC-HRMS analyses of intracellular metabolites (G6P, G1P, and UDP-Glc) collected from the indicated cell lines incubated in glucose-rich SDM79 medium. Only G6P, G1P, and UDP-Glc are shown in this figure; for other hexose phosphates and triose phosphates, see Fig. S6. nd, nondetectable. 
enzymes being involved in glycogenesis and cell wall glucan synthesis, respectively (21). All of these biosynthetic pathways require glycosyltransferases, which have not been detected in the glycosomal proteomes $(46,47)$ or in the repertoire of PTS-containing proteins (48). This supports the view that UDP-Glc and UDP-Gal are not produced in the glycosomes to feed glycosylation reactions inside glycosomes. Alternatively, glycosomal UDP-Glc may have a signaling role, as previously observed in animals and plants $(49,50)$.

Piggybacking is a low-efficiency import process, as observed for UGP. Piggybacking has been described as an import mechanism with relatively low efficiency in four out of five examples of physiological hetero-oligomer import into peroxisomes reported so far, i.e., superoxide dismutase (SOD1) (25) and lactate dehydrogenase (LDH) (26) in mammals and pyrazinamidase/nicotinamidase (PNC1) (29) and malate dehydrogenase 2 (Mdh2) (28) in yeast, which are coimported with the PTS-containing copper chaperone SOD1 (CCS), readthrough-extended LDH (LDHBx), glycerol-3-phosphate dehydrogenase (GPD1), and Mdh3, respectively. These four coimported proteins display dual peroxisomal and cytosolic localizations, with the majority remaining within the cytosol $(28,51)$. Similarly, approximately half of UGP remains in the cytosol. The reason for this relatively low import efficiency has been elucidated by the demonstration that the PST1 receptor (PEX5), required for peroxisomal import of PTS1-containing proteins, binds preferentially to monomers rather than to oligomers (52). Interestingly, weak protein-protein interactions are sufficient to support piggyback import. Indeed, blue native gels failed to show an interaction between the mammalian SOD and CCS partners (25), and synthetic substrates designed to evaluate the import of proteins showed dissociation constants $\left(K_{d}\right)$ differing by over 3 orders of magnitude, with even an apparent $K_{d}$ of $\sim 6 \times 10^{-3} \mathrm{M}$ allowing the detection of piggyback import (53). Despite several attempts, we did not observe any interaction between UGP and PEPCK using coimmunoprecipitation or native gels, suggesting that these interactions are weak and transient. In agreement with this weak interaction, PEPCK is in large excess compared to UGP, as illustrated by the $\sim 30$-fold-higher enzymatic activity of PEPCK than of UGP (670 versus $20 \mathrm{mU} \cdot \mathrm{mg}^{-1}$ of protein) (54) and the $\sim 100$-foldhigher peptide counts for PEPCK than for UGP in proteomics analyses of glycosomal fractions from PCFs (see the PXD020190 data set in the PRIDE partner repository). In conclusion, our results support the role of hetero-oligomer import by piggybacking as an alternative route for import of glycosomal proteins, as described for peroxisomes of mammals and yeast. More importantly, the UGP/PEPCK association provides the first example of hetero-oligomeric import by piggybacking involving two proteins not functionally related, since PEPCK is involved in the maintenance of glycosomal redox and ATP/ADP balances, as well as gluconeogenesis $(30,35)$. Indeed, among the other known examples of piggybacking, CCS is the chaperone of SOD1 (25), LDH and LDHBx are encoded by the same gene (26), Mdh2 and Mdh3 are Mdh isoforms (28), and the PST1-containing phosphatase $B$ subunit and phosphatases $A / C$ subunits form an heterotrimeric enzymatic complex (27); however, the peroxisomal functions of PNC1 and GPD1 are unknown (29).

UGP and PEPCK interact only transiently upon their import into newly produced import-competent glycosomes. Since the formation of the UGP/PEPCK heterodimer may occur mainly during UGP import into the organelle, the analysis of UGP/PEPCK interactions using the PLA approach provides new insights into glycosomal import of proteins and multiplication of the organelles. In mammalian cells, peroxisomes multiply by the de novo ER route and by growth and division. The latter case involves an asymmetric process generating new peroxisomes via formation of a membrane compartment and subsequent import of newly synthesized matrix proteins (55-57). Indeed, overexpression of the membrane peroxin $\operatorname{Pex} 11 \mathrm{p} \beta$ resulted in the formation in mammalian cells of preperoxisomal membrane structures composed of mature globular domains and tubular extensions, the latter being maturated by import of matrix proteins (56). Equivalent clusters of tubular glycosomal membranes were also observed by overexpressing Pex11 in T. brucei (58), and clusters of elongated 
glycosomes have more recently been observed in BSF trypanosomes by whole-cell reconstruction using three-dimensional (3D) electron microscopy (59). In addition, $T$. brucei expresses Fis1 and Dpl1, two key proteins involved in the fission of newly produced peroxisomes in other eukaryotes (60-62). Overall, these observations confirm that glycosomes multiply by growth and division, as observed for the mammalian peroxisomes. This also implies that the new peroxisomes/glycosomes produced by growth and division are the most competent organelles for protein import and that they represent only a limited fraction of the organelle population, supporting the heterogeneity observed before among the peroxisomal (63) and glycosomal (33) populations. We thus propose that the structures showing close UGP/PEPCK proximity by PLA correspond to newly produced import-competent glycosomes. Considering that (i) PEPCK and UGP physically interact mainly during import at the glycosomal membrane because of their weak and transient interaction, (ii) that only up to 10 dots per cell correspond to physical proximity between PEPCK and UGP, with most cells containing 2 to 5 dots (Fig. 2), while PEPCK and UGP appear localized in almost all, if not all, glycosomes (Fig. 1E and Fig. S1), and (iii) that the number of glycosomes was estimated to be 60 to 65 per $G_{1}$ trypanosome cell $(59,64)$, one could consider that the 3 to $10 \%$ of the organelles showing UGP/PEPCK interaction by PLA are newly produced glycosomes importing the matrix proteins, including PEPCK and UGP, in this context.

\section{MATERIALS AND METHODS}

Trypanosomes and cell cultures. The procyclic form of T. brucei EATRO1125.T7T (TetR-HYG T7RNAPOL-NEO, where TetR stands for tetracycline resistance, HYG is hygromycin, POL is polymerase, and NEO is neomycin) was cultured at $27^{\circ} \mathrm{C}$ in SDM79 medium containing $10 \%$ (vol/vol) heat-inactivated fetal calf serum, $5 \mu \mathrm{g} \cdot \mathrm{ml}^{-1}$ hemin (65), $25 \mu \mathrm{g} \cdot \mathrm{ml}^{-1}$ hygromycin, and $10 \mu \mathrm{g} \cdot \mathrm{ml}^{-1}$ neomycin. Alternatively, the cells were cultivated in a glucose-free medium derived from SDM79, called SDM79GlcFree (37). The bloodstream form of T. brucei 427 90-13 (TetR-HYG T7RNAPOL-NEO) was cultured at $37^{\circ} \mathrm{C}$ in Iscove's modified Dulbecco's medium (IMDM) supplemented with $10 \%$ (vol/vol) heat-inactivated fetal calf serum (FCS), $0.25 \mathrm{mM} \beta$-mercaptoethanol, $36 \mathrm{mM} \mathrm{NaHCO}{ }_{3}, 1 \mathrm{mM}$ hypoxanthine, $0.16 \mathrm{mM}$ thymidine, $1 \mathrm{mM}$ sodium pyruvate, $0.05 \mathrm{mM}$ bathocuproine, and $2 \mathrm{mM}$ L-cysteine (66). Cells were transfected as previously described (67). Overexpression and RNAi cell lines were induced with tetracycline $\left(1 \mu \mathrm{g} \cdot \mathrm{ml}^{-1}\right)$. Growth was monitored by daily cell counting with the cytometer Guava EasyCyte.

Expression of MYC-tagged UGP, TY-tagged UGP, eGFP-PEPCK truncations, and TY-tagged PEPCK. The UGP gene (Tb927.10.13130) was cloned using the In-Fusion cloning system (Clontech) at the HindIII-Ndel restriction sites of pLew100-X-MYC, which was designed for expression of recombinant protein tagged at the C-terminal extremity with 3 MYC epitopes (modified from reference 68). The EATRO1125.T7T parental cell line and the $\Delta$ pepck (30), TY-PEPCK, and $\triangle$ pepck/TY-PEPCK cell lines were transfected with the pLew100-UGP-MYC tetracycline-inducible plasmid, and cells were selected in SDM79 containing phleomycin $\left(5 \mu \mathrm{g} \cdot \mathrm{ml}^{-1}\right)$. The UGP gene was also in situ tagged at the $\mathrm{N}$-terminal or C-terminal extremity, as previously described (69). The TY-UGP gene is flanked by the aldolase $5^{\prime}$ untranscribed region (5'UTR) and the UGP $3^{\prime}$ UTR, while the UGP-TY gene is flanked by the UGP $5^{\prime}$ UTR and the aldolase $3^{\prime}$ UTR. Briefly, the DNA sequence encoding $10 \times$ TY1 tag and blasticidin (BLA) resistance cassette was amplified from the pPOTv7-10 $\times$ TY1 vector using long primers (see Table S1 in the supplemental material) that incorporate a $5^{\prime}$ overhang of 80 nucleotides ( $n t$ ) homologous to the UGP gene and its UTR. For the production of truncated UGP versions tagged with $10 \times$ TY1 at their C-terminal extremity, the forward primers were designed within the UGP gene extension to produce proteins containing the first 66 ( 1 to 66 ), 124 ( 1 to 124 ), 173 ( 1 to 173 ), and 226 ( 1 to 226 ) N-terminal residues. The PCR products were precipitated with ethanol before being used for transfection, and cells were selected in SDM79 containing blasticidin $\left(20 \mu \mathrm{g} \cdot \mathrm{ml}^{-1}\right)$. We also expressed truncated versions of a recoded UGP (rUGP) (Fig. S7) lacking either the first 66 or the first 124 residues fused to the $10 \times$ TY1 tag at their C-terminal extremity. The PCR fragments corresponding to a complete or truncated rUGP gene fused to the TY tag and blasticidin cassette from pPOTv7 were obtained by overlapping PCR and cloned into pGEM-T. Cells were transfected with $10 \mu \mathrm{g}$ of plasmid digested with Notl. For expression of truncated eGFP-PEPCK versions, the $\Delta$ pepck cell line (30) was transfected with the pLew100 tetracycline-inducible plasmid containing truncated versions of PEPCK fused at the N-terminal extremity to eGFP to increase the stability of the truncated recombinant proteins. PCR fragments corresponding to the truncations of PEPCK at residues 140, 180, 214, and 321 were inserted between the Xhol and Xbal restriction sites of the pLew100eGFPX plasmid using the In-Fusion cloning system (Clontech). The PEPCK gene was also in situ tagged at the $\mathrm{N}$ terminus, as described above, with the TY-PEPCK gene flanked by the aldolase $5^{\prime} \mathrm{UTR}$ and the PEPCK 3'UTR.

Production of recombinant glycosomal UGP proteins. To target UGP exclusively to the glycosomes, the recoded recombinant UGP (rUGP) (Fig. S7) gene was inserted in the pHD1336 expression vector (42). For this purpose, the rUGP was fused at its C-terminal extremity to a $3 \times \mathrm{MYC}$ tag followed by (i) the sequence encoding the last $12 \mathrm{C}$-terminal residues of the glycosomal fumarate reductase $(F R D g)$ 
gene, which contains a PTS1 (rUGP-FRDgPST1), (ii) the full-length PTS1-containing glycosomal phosphoglycerate $(P G K C)$ gene (rUGP-PGKc*), and (iii) the full-length PTS1-containing glycerol-3-phosphate dehydrogenase (GPDH) gene (rUGP-GPDH). The K215 residue, essential for PGK activity (36), was replaced by alanine. In order to increase the net charge of residues at the $C$ terminus, which is a major determinant of peroxisomal import efficiency (70), we modified one residue in the C-terminal extremity of PGK (TLRNRW-SSL instead of TLSNRW-SSL) and of GPDH (PARPRT-SKM instead of PALPRT-SKM). The pHD1336-rUGP-FRDgPST1 plasmid, provided by the GeneCust Company, was used for cloning the synthesized genes (GeneCust) PGKc* and GPDH in the Mlul-BamHI restriction sites. The EATRO1125.T7T parental cell line was transfected, and cells were selected in SDM79 containing blasticidin $\left(20 \mu \mathrm{g} \cdot \mathrm{ml}^{-1}\right)$.

Inhibition of UGP gene expression. The inhibition of UGP expression by RNAi was achieved by expression of stem-loop "sense/antisense" RNA molecules targeting a 537-bp fragment of the UGP gene introduced into the pLew100 tetracycline-inducible expression vector. A PCR-amplified 579-bp fragment, containing the antisense UGP sequence was inserted between HindIII and BamHI restriction sites of the pLew100 plasmid. Then, the separate 537-bp PCR-amplified fragment containing the sense UGP sequence was inserted upstream of the antisense sequence, using HindIII and Xhol restriction sites. The resulting plasmid, pLew-UGP-SAS, contains a sense and antisense version of the UGP fragment separated by a 42-bp fragment. The ${ }^{R N A}$ UGP and ${ }^{R N A}$ UGP/EXP rUGP-GPDH mutants were generated by transfecting the EATRO1125.T7T and ${ }^{R N A}$ UGP cell lines with the pLew-UGP-SAS plasmid and the pHD1336rUGP-GPDH plasmid, respectively. Transfected cells were selected in SDM79 medium containing hygromycin $\left(25 \mu \mathrm{g} \cdot \mathrm{ml}^{-1}\right)$, neomycin $\left(10 \mu \mathrm{g} \cdot \mathrm{ml}^{-1}\right)$, and phleomycin $\left(5 \mu \mathrm{g} \cdot \mathrm{ml}^{-1}\right)$, with addition of blasticidin $\left(20 \mu \mathrm{g} \cdot \mathrm{ml}^{-1}\right)$ for the ${ }^{\text {RNAiUGP/EXP }}$ rUGP-GPDH cell line.

Production of UGP null mutants. Replacement of the UGP gene by the phleomycin and puromycin resistance markers via homologous recombination was performed with DNA fragments containing the resistance marker gene flanked by the UTR sequences. Briefly, an Hpal DNA fragment containing the PAC or $B L E$ resistance marker gene preceded by the UGP 5'UTR fragment (522 bp) and followed by the UGP 3'UTR fragment (526 bp) was cloned into the PGEM-T plasmid. The UGP knockout mutants were generated in the ${ }^{\text {EXP }}$ rUGP-GPDH and ${ }^{\text {EXP }}$ rUGP cell lines in the presence of tetracycline. The ${ }^{\text {EXP }}$ rUGP cell line was generated by transfecting the EATRO1125.T7T parental cell line with the pHD1336 vector expressing the rUGP sequence followed by a MYC tag sequence under the control of tetracycline. Transfected cells were selected in SDM79 medium containing blasticidin $\left(20 \mu \mathrm{g} \cdot \mathrm{ml}^{-1}\right)$, phleomycin $\left(5 \mu \mathrm{g} \cdot \mathrm{ml}^{-1}\right)$, puromycin $\left(1 \mu \mathrm{g} \cdot \mathrm{ml}^{-1}\right)$, and tetracycline $\left(1 \mu \mathrm{g} \cdot \mathrm{ml}^{-1}\right)$. The selected cell lines rUGP::GPDH ${ }^{T i}-B L A$ TetR-HYG T7RNAPOL-NEO $\triangle$ ugp::PAC/

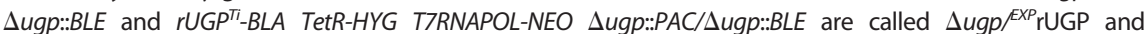
$\Delta u g p /{ }^{E X P}$ rUGP-GPDH, respectively.

Preparation of glycosomal and cytosolic fractions. Cell homogenates were obtained by grinding prewashed cells with silicon carbide (200 mesh) in STE buffer ( 25 mM Tris, 1 mM EDTA, 250 mM sucrose, $\mathrm{pH}$ 7.8) (71) supplemented with the Complete EDTA-free protease inhibitor cocktail (Roche). The cells were microscopically checked for at least $90 \%$ disruption. The lysates were diluted in $7 \mathrm{ml}$ of STE and centrifuged at $1,000 \times g$ and then at $5,000 \times g$ for $10 \mathrm{~min}$ each time at $4^{\circ} \mathrm{C}$. The supernatants were centrifuged at $42,000 \times g$ for $10 \mathrm{~min}$ at $4^{\circ} \mathrm{C}$ to yield the glycosome-enriched pellets and the cytosolic fractions (supernatants). The glycosomal pellets were washed once with $1 \mathrm{ml}$ of STE before centrifugation at $42,000 \times g$ for $10 \mathrm{~min}$ at $4^{\circ} \mathrm{C}$ and resuspension in $0.2 \mathrm{ml}$ of STE. Equivalent amounts of protein from glycosomal and cytosolic fractions were analyzed by Western blotting.

Digitonin permeabilization. Trypanosomes were washed two times in cold phosphate-buffered saline (PBS) and resuspended at $10 \mathrm{mg}$ of protein per $\mathrm{ml}$ in STE buffer supplemented with $150 \mathrm{mM} \mathrm{NaCl}$ and protease inhibitors. Cell aliquots $(100 \mu \mathrm{l})$ were incubated with increasing quantities of digitonin (Sigma) for $4 \mathrm{~min}$ at $25^{\circ} \mathrm{C}$, before centrifugation at $14,000 \times g$ for $2 \mathrm{~min}$. The supernatants were analyzed by Western blotting.

Cell fractionation by hypotonic lysis. BSF and PCF parasites $\left(2 \cdot 10^{8}\right)$ were washed in PBS and hypotonically lysed in the presence of protease inhibitors by incubating them in $5 \mathrm{mM} \mathrm{Na}_{2} \mathrm{HPO}_{4}, 0.3 \mathrm{mM}$ $\mathrm{KH}_{2} \mathrm{PO}_{4}$ for $30 \mathrm{~min}$ at $4^{\circ} \mathrm{C}$ before centrifugation at $14,000 \times \mathrm{g}$ for $15 \mathrm{~min}$. The pellet was solubilized in $2 \%$ SDS, and both the supernatant and the pellet were analyzed by Western blotting.

Immunofluorescence and proximity ligation assay (PLA). Cells were washed twice with PBS and then treated (+) or not treated (-) with $0.04 \mathrm{mg}$ of digitonin per $\mathrm{mg}$ of protein for $4 \mathrm{~min}$ at $25^{\circ} \mathrm{C}$. After centrifugation at $14,000 \times g$ for 2 min and being washed, the cellular pellets were resuspended in PBS and fixed with $4 \%$ paraformaldehyde (PFA) for $10 \mathrm{~min}$ at room temperature. The cells were spread on poly-L-lysine-coated slides and permeabilized with $0.05 \%$ Triton X-100. After incubation in PBS containing $4 \%$ bovine serum albumin (BSA) overnight, cells were incubated for 45 min with primary antibodies (Table S2), washed with PBS, and incubated for 45 min with secondary antibodies (Table S2). Slides were washed and mounted with SlowFade Gold (Molecular Probes). Images were acquired with MetaMorph software on a Zeiss Imager Z1 or Axioplan 2 microscope as previously described (72).

In situ PLA was performed using the Duolink In Situ Red mouse/rabbit starter kit (Sigma-Aldrich) according to the manufacturer's recommendations. Briefly, PFA-fixed and Triton X-100-permeabilized cells were spread on slides as described above. The cells were blocked with Duolink blocking solution for $60 \mathrm{~min}$ at $37^{\circ} \mathrm{C}$. Primary rabbit anti-MYC $(1 / 1,000)$ and mouse anti-TY1 $(1 / 5,000)$ antibodies were diluted in Duolink antibody diluent and incubated for $60 \mathrm{~min}$ at room temperature. The slides were washed for $10 \mathrm{~min}$ in wash buffer A and incubated with the PLUS and MINUS PLA probes for $60 \mathrm{~min}$ at $37^{\circ} \mathrm{C}$. Ligation and amplification steps were performed according to the manufacturer's instructions. After being washed, cells were blocked with PBS-4\% BSA overnight. The cells where counterstained with mouse anti-PPDK ( $\alpha$ PPDK) (67). Slides were mounted in Duolink in situ mounting medium with DAPI 
(4',6-diamidino-2-phenylindole). Images were acquired as described for immunofluorescence and analyzed using ImageJ. Cells were counted manually using cell counter ImageJ plugin.

BN-PAGE. Cells $\left(10^{8}\right)$ were washed in PBS and resuspended in SoTE $(0.6 \mathrm{M}$ sorbitol, $2 \mathrm{mM}$ EDTA, $20 \mathrm{mM}$ Tris- $\mathrm{HCl}, \mathrm{pH} 7.5$ ) (73). Cells were incubated with 0 or $0.16 \mathrm{mg}$ of digitonin per mg of protein for $4 \mathrm{~min}$ at $25^{\circ} \mathrm{C}$ before centrifugation at $14,000 \times g$ for $2 \mathrm{~min}$. The supernatants containing both the cytosolic and glycosomal proteins were analyzed by blue native PAGE (BN-PAGE) on a precast ( 3 to $12 \%$ ) BisTris polyacrylamide gel (Invitrogen) according to standard methods.

Western blot analyses. Total protein extracts $\left(5 \cdot 10^{6}\right.$ cells), glycosomal and cytosolic fractions, or supernatants obtained after digitonin treatment were separated by SDS-PAGE (10\%) and immunoblotted on Trans-Blot Turbo midi size polyvinylidene difluoride (PVDF) membranes (Bio-Rad) (74). Immunodetection was performed as described previously $(74,75)$ using the primary antibodies and conditions summarized in Table S2. Revelation was performed using the Clarity Western enhanced-chemiluminescence (ECL) substrate as described by the manufacturer (Bio-Rad). Images were acquired and analyzed with the ImageQuant LAS 4000 luminescent image analyzer.

UGP activity assay. The UGP activity in total lysates and aliquots of glycosomal and cytosolic fractions was measured as previously described (22). For normalization of the UGP activities, the malic enzyme activity was determined on the total cell extracts and the cytosolic fractions, as described before (76). For normalization of the UGP activities in glycosomal extracts, the glycerol kinase activity was determined as described before (77). The PEPCK activity was measured in total lysates as previously described (54).

Label-free quantitative proteomics. Enriched glycosomal fractions were loaded on a $10 \%$ acrylamide SDS-PAGE gel, and proteins were visualized by colloidal blue staining. The steps of sample preparation, protein digestion, and liquid chromatography-mass spectrometry (LC-MS) parameters used for nanoscale LC-tandem MS (nanoLC-MS/MS) analysis on a Q-Exactive mass spectrometer were previously described (78). For protein identification, the SEQUEST HT and Mascot 2.4 algorithms through Proteome Discoverer 1.4 software (Thermo Fisher Scientific Inc.) were used for protein identification in batch mode by searching against a Trypanosoma brucei protein database $(11,119$ entries, release 46$)$. This database was downloaded from the http://tritrypdb.org website. Two missed enzyme cleavages were allowed. Mass tolerances in MS and MS/MS were set to $10 \mathrm{ppm}$ and $0.02 \mathrm{Da}$. The oxidation of methionine, acetylation of lysine, and deamidation of asparagine and glutamine were searched as dynamic modifications. Carbamidomethylation on cysteine was searched as a static modification. Peptide validation was performed using the Percolator algorithm (79), and only "high-confidence" peptides corresponding to a $1 \%$ false-discovery rate (FDR) at the peptide level were retained. Raw LC-MS/MS data were imported in Progenesis QI (version 2.0; Nonlinear Dynamics, a Waters Company) for feature detection, alignment, and quantification. All sample features were aligned according to retention times by manually inserting up to 50 landmarks, followed by automatic alignment, to maximally lay over all the two-dimensional $(\mathrm{m} / \mathrm{z}$ and retention time) feature maps. Singly charged ions and ions with a higher charge states than 6 were excluded from analysis. All remaining features were used to calculate a normalization factor for each sample that corrects for experimental variation. Peptide identifications (with an FDR of $<1 \%$ ) were imported into Progenesis. Only nonconflicting features and unique peptides were considered for calculation of quantification at the protein level. A minimum of two peptides matched to a protein was used as the criterion for identification as a differentially expressed protein.

MS analyses of intracellular metabolites by IC-HRMS. Parental and mutant cell lines grown in SDM79 medium were collected on filters by fast filtration preparation $\left(2 \cdot 10^{7}\right.$ cells per filter), as described before (30). Metabolites were analyzed by liquid anion exchange chromatography on a Dionex ICS-5000 + reagent-free HPIC (Thermo Fisher Scientific, Sunnyvale, CA, USA) system coupled with a Thermo Scientific linear trap quadrupole (LTQ) Orbitrap Velos hybrid Fourier-transform (FT) mass spectrometer (FTMS; Thermo Fisher Scientific, San Jose, CA, USA). The metabolites were separated within 48 min using a linear gradient elution of $\mathrm{KOH}$ applied to an lonPac AS11 column ( 250 by $2 \mathrm{~mm}$; Dionex) equipped with an AG11 guard column ( 50 by $2 \mathrm{~mm}$; Dionex) at a flow rate of $0.35 \mathrm{ml} \cdot \mathrm{min}^{-1}$. The column and autosampler temperature were $30^{\circ} \mathrm{C}$ and $4^{\circ} \mathrm{C}$, respectively. The injected sample volume was $15 \mu \mathrm{l}$. Mass detection was carried out in the negative electrospray ionization (ESI) mode. The settings of the mass spectrometer were as follows: spray voltage was at $2.7 \mathrm{kV}$, capillary and desolvation temperatures were 350 and $350^{\circ} \mathrm{C}$, respectively, and the maximum injection time was $50 \mathrm{~ms}$. Nitrogen was used as the sheath gas (pressure, 50 units) and auxiliary gas (pressure, 5 units). The automatic gain control (AGC) was set at 1E6 for full-scan mode, with a mass resolution of 60,000 (at $400 \mathrm{~m} / \mathrm{z}$ ). Data acquisition was performed using Thermo Scientific Xcalibur software. The identification of metabolites relied upon matching accurate masses from an FTMS scan (mass tolerance of $5 \mathrm{ppm}$ ) with retention time using TraceFinder 3.2 software. The absolute levels of intracellular metabolites were quantified based on the isotope dilution mass spectrometry (IDMS) approach.

Data availability. The mass spectrometry proteomics data have been deposited in the ProteomeXchange Consortium (http://proteomecentral.proteomexchange.org) via the PRIDE partner repository (80) with the data set identifier PXD020190.

\section{SUPPLEMENTAL MATERIAL}

Supplemental material is available online only.

FIG S1, TIF file, 0.3 MB.

FIG S2, TIF file, 0.9 MB.

FIG S3, TIF file, 0.7 MB. 
FIG S4, TIF file, 0.4 MB.

FIG S5, TIF file, 0.2 MB.

FIG S6, TIF file, 0.9 MB.

FIG S7, TIF file, 0.7 MB.

TABLE S1, DOCX file, $0.1 \mathrm{MB}$.

TABLE S2, DOCX file, 0.1 MB.

\section{ACKNOWLEDGMENTS}

We thank Paul A. Michels (Edinburgh, Scotland) for providing us with the antienolase and antialdolase immune sera, as well as Keith Gull (Oxford, United Kingdom) and Klaus Ersfeld (Hull, United Kingdom) for providing us with the anti-TY (BB2) and anti-MYC (9E10) monoclonal antibodies, respectively.

F.B.'s group was supported by the Université de Bordeaux, the Centre National de la Recherche Scientifique (CNRS), and the Agence Nationale de la Recherche (ANR) through GlycoNov grants of the ANR-BLANC-2015 call (grant ANR-15-CE15-0025-01) and the French Government (ANR; the Investissement d'Avenir program and the Laboratoire d'Excellence [LabEx]) through the LabEx ParaFrap (French Parasitology Alliance for Health Care) (grant ANR-11-LABX-0024-PARAFRAP). MetaboHub-MetaToul (Metabolomics \& Fluxomics' facilities, Toulouse, France, http://www.metatoul.fr) is supported by the ANR grant MetaboHUB-ANR-11-INBS-0010.

\section{REFERENCES}

1. Buscher P, Cecchi G, Jamonneau V, Priotto G. 2017. Human African trypanosomiasis. Lancet 390:2397-2409. https://doi.org/10.1016/S0140 -6736(17)31510-6.

2. Bringaud F, Riviere L, Coustou V. 2006. Energy metabolism of trypanosomatids: adaptation to available carbon sources. Mol Biochem Parasitol 149:1-9. https://doi.org/10.1016/j.molbiopara.2006.03.017.

3. Opperdoes FR, Borst P, Spits H. 1977. Particle-bound enzymes in the bloodstream form of Trypanosoma brucei. Eur J Biochem 76:21-28. https://doi.org/10.1111/j.1432-1033.1977.tb11566.x.

4. Allmann S, Bringaud F. 2017. Glycosomes: a comprehensive view of their metabolic roles in T. brucei. Int J Biochem Cell Biol 85:85-90. https://doi .org/10.1016/j.biocel.2017.01.015.

5. Bard F, Chia J. 2016. Cracking the glycome encoder: signaling, trafficking, and glycosylation. Trends Cell Biol 26:379-388. https://doi.org/10.1016/j .tcb.2015.12.004.

6. Bandini G, Marino K, Guther ML, Wernimont AK, Kuettel S, Qiu W, Afzal S, Kelner A, Hui R, Ferguson MA. 2012. Phosphoglucomutase is absent in Trypanosoma brucei and redundantly substituted by phosphomannomutase and phospho-N-acetylglucosamine mutase. Mol Microbiol 85:513-534. https://doi.org/10.1111/j.1365-2958.2012.08124.x.

7. Marino K, Guther ML, Wernimont AK, Amani M, Hui R, Ferguson MA. 2010. Identification, subcellular localization, biochemical properties, and high-resolution crystal structure of Trypanosoma brucei UDP-glucose pyrophosphorylase. Glycobiology 20:1619-1630. https://doi.org/ 10.1093/glycob/cwq115.

8. Marino K, Guther ML, Wernimont AK, Qiu W, Hui R, Ferguson MA. 2011. Characterization, localization, essentiality, and high-resolution crystal structure of glucosamine 6-phosphate $\mathrm{N}$-acetyltransferase from Trypanosoma brucei. Eukaryot Cell 10:985-997. https://doi.org/10.1128/EC.05025-11.

9. Roper JR, Guther ML, Milne KG, Ferguson MA. 2002. Galactose metabolism is essential for the African sleeping sickness parasite Trypanosoma brucei. Proc Natl Acad Sci U S A 99:5884-5889. https://doi.org/10.1073/pnas 092669999 .

10. Stokes MJ, Guther ML, Turnock DC, Prescott AR, Martin KL, Alphey MS, Ferguson MA. 2008. The synthesis of UDP-N-acetylglucosamine is essential for bloodstream form Trypanosoma brucei in vitro and in vivo and UDP-N-acetylglucosamine starvation reveals a hierarchy in parasite protein glycosylation. J Biol Chem 283:16147-16161. https://doi.org/10 .1074/jbc.M709581200.

11. Turnock DC, Izquierdo L, Ferguson MA. 2007. The de novo synthesis of GDP-fucose is essential for flagellar adhesion and cell growth in Trypanosoma brucei. J Biol Chem 282:28853-28863. https://doi.org/10.1074/jbc M704742200.
12. Ferguson MA. 1997. The surface glycoconjugates of trypanosomatid parasites. Philos Trans R Soc Lond B 352:1295-1302. https://doi.org/10.1098/ rstb.1997.0113.

13. Decker D, Kleczkowski LA. 2018. UDP-sugar producing pyrophosphorylases: distinct and essential enzymes with overlapping substrate specificities, providing de novo precursors for glycosylation reactions. Front Plant Sci 9:1822. https://doi.org/10.3389/fpls.2018.01822.

14. Sousa M, Parodi AJ. 1995. The molecular basis for the recognition of misfolded glycoproteins by the UDP-Glc:glycoprotein glucosyltransferase. EMBO J 14:4196-4203. https://doi.org/10.1002/j.1460-2075.1995.tb00093.x.

15. Tetaud E, Barrett MP, Bringaud F, Baltz T. 1997. Kinetoplastid glucose transporters. Biochem J 325:569-580. https://doi.org/10.1042/bj3250569.

16. Damerow S, Hoppe C, Bandini G, Zarnovican P, Buettner FFR, Buettner FR, Lüder CGK, Ferguson MAJ, Routier FH. 2015. Depletion of UDP-glucose and UDP-galactose using a Degron system leads to growth cessation of Leishmania major. PLoS Negl Trop Dis 9:e0004205. https://doi.org/10 .1371/journal.pntd.0004205.

17. Fuhring Jl, Cramer JT, Schneider J, Baruch P, Gerardy-Schahn R, Fedorov R. 2015. A quaternary mechanism enables the complex biological functions of octameric human UDP-glucose pyrophosphorylase, a key enzyme in cell metabolism. Sci Rep 5:9618. https://doi.org/10.1038/srep09618.

18. Ebrecht AC, Asencion Diez MD, Piattoni CV, Guerrero SA, Iglesias AA. 2015. The UDP-glucose pyrophosphorylase from Giardia lamblia is redox regulated and exhibits promiscuity to use galactose-1-phosphate. Biochim Biophys Acta 1850:88-96. https://doi.org/10.1016/j.bbagen.2014.10.002.

19. Martinez LI, Piattoni CV, Garay SA, Rodrigues DE, Guerrero SA, Iglesias AA. 2011. Redox regulation of UDP-glucose pyrophosphorylase from Entamoeba histolytica. Biochimie 93:260-268. https://doi.org/10.1016/j.biochi 2010.09.019.

20. Muchut RJ, Calloni RD, Herrera FE, Garay SA, Arias DG, Iglesias AA, Guerrero SA. 2018. Elucidating paramylon and other carbohydrate metabolism in Euglena gracilis: kinetic characterization, structure and cellular localization of UDP-glucose pyrophosphorylase. Biochimie 154:176-186. https://doi.org/10.1016/j.biochi.2018.09.006.

21. Smith TL, Rutter J. 2007. Regulation of glucose partitioning by PAS kinase and Ugp1 phosphorylation. Mol Cell 26:491-499. https://doi.org/10.1016/ j.molcel.2007.03.025.

22. Lamerz AC, Haselhorst T, Bergfeld AK, von Itzstein M, Gerardy-Schahn R. 2006. Molecular cloning of the Leishmania major UDP-glucose pyrophosphorylase, functional characterization, and ligand binding analyses using NMR spectroscopy. J Biol Chem 281:16314-16322. https://doi.org/10 $.1074 /$ jbc.M600076200. 
23. Sampaio Guther ML, Prescott AR, Kuettel S, Tinti M, Ferguson MAJ. 2021 Nucleotide sugar biosynthesis occurs in the glycosomes of procyclic and bloodstream form Trypanosoma brucei. PLoS Negl Trop Dis 15:e0009132. https://doi.org/10.1371/journal.pntd.0009132.

24. Rucktaschel R, Girzalsky W, Erdmann R. 2011. Protein import machineries of peroxisomes. Biochim Biophys Acta 1808:892-900. https://doi.org/10 .1016/j.bbamem.2010.07.020.

25. Islinger M, Li KW, Seitz J, Volkl A, Luers GH. 2009. Hitchhiking of Cu/Zn superoxide dismutase to peroxisomes-evidence for a natural piggyback import mechanism in mammals. Traffic 10:1711-1721. https://doi.org/10 $.1111 / \mathrm{j} .1600-0854.2009 .00966 . x$.

26. Schueren F, Lingner T, George R, Hofhuis J, Dickel C, Gartner J, Thoms S. 2014. Peroxisomal lactate dehydrogenase is generated by translational readthrough in mammals. Elife 3:e03640. https://doi.org/10.7554/eLife .03640 .

27. Kataya AR, Heidari B, Hagen L, Kommedal R, Slupphaug G, Lillo C. 2015. Protein phosphatase 2A holoenzyme is targeted to peroxisomes by piggybacking and positively affects peroxisomal beta-oxidation. Plant Physiol 167:493-506. https://doi.org/10.1104/pp.114.254409.

28. Gabay-Maskit S, Cruz-Zaragoza LD, Shai N, Eisenstein M, Bibi C, Cohen N, Hansen T, Yifrach E, Harpaz N, Belostotsky R, Schliebs W, Schuldiner M, Erdmann R, Zalckvar E. 2020. A piggybacking mechanism enables peroxisomal localization of the glyoxylate cycle enzyme Mdh2 in yeast. J Cell Sci 133:jcs244376. https://doi.org/10.1242/jcs.244376.

29. Effelsberg D, Cruz-Zaragoza LD, Tonillo J, Schliebs W, Erdmann R. 2015. Role of Pex21p for piggyback import of Gpd1p and Pnc1p into peroxisomes of Saccharomyces cerevisiae. J Biol Chem 290:25333-25342. https://doi.org/10.1074/jbc.M115.653451.

30. Ebikeme C, Hubert J, Biran M, Gouspillou G, Morand P, Plazolles N, Guegan F, Diolez P, Franconi JM, Portais JC, Bringaud F. 2010. Ablation of succinate production from glucose metabolism in the procyclic trypanosomes induces metabolic switches to the glycerol 3-phosphate/dihydroxyacetone phosphate shuttle and to proline metabolism. J Biol Chem 285:32312-32324. https://doi.org/10.1074/jbc.M110.124917.

31. Walter T, Erdmann R. 2019. Current advances in protein import into peroxisomes. Protein J 38:351-362. https://doi.org/10.1007/s10930-019-09835-6.

32. Soderberg $O$, Gullberg $M$, Jarvius $M$, Ridderstrale $K$, Leuchowius $K J$, Jarvius J, Wester K, Hydbring P, Bahram F, Larsson LG, Landegren U. 2006. Direct observation of individual endogenous protein complexes in situ by proximity ligation. Nat Methods 3:995-1000. https://doi.org/10.1038/ nmeth947.

33. Gualdron-Lopez M, Chevalier N, Van Der Smissen P, Courtoy PJ, Rigden DJ, Michels PAM. 2013. Ubiquitination of the glycosomal matrix protein receptor PEX5 in Trypanosoma brucei by PEX4 displays novel features. Biochim Biophys Acta 1833:3076-3092. https://doi.org/10.1016/j.bbamcr .2013.08.008.

34. Trapani S, Linss J, Goldenberg S, Fischer H, Craievich AF, Oliva G. 2001. Crystal structure of the dimeric phosphoenolpyruvate carboxykinase (PEPCK) from Trypanosoma cruzi at 2 A resolution. J Mol Biol 313:1059-1072. https:// doi.org/10.1006/jmbi.2001.5093.

35. Deramchia K, Morand P, Biran M, Millerioux $Y$, Mazet M, Wargnies $M$, Franconi JM, Bringaud F. 2014. Contribution of pyruvate phosphate dikinase in the maintenance of the glycosomal ATP/ADP balance in the Trypanosoma brucei procyclic form. J Biol Chem 289:17365-17378. https://doi .org/10.1074/jbc.M114.567230.

36. Bernstein BE, Hol WG. 1998. Crystal structures of substrates and products bound to the phosphoglycerate kinase active site reveal the catalytic mechanism. Biochemistry (Mosc) 37:4429-4436. https://doi.org/10.1021/ bi9724117.

37. Wargnies M, Bertiaux E, Cahoreau E, Ziebart N, Crouzols A, Morand P, Biran M, Allmann S, Hubert J, Villafraz O, Millerioux Y, Plazolles N, Asencio C, Riviere L, Rotureau B, Boshart M, Portais JC, Bringaud F. 2018. Gluconeogenesis is essential for trypanosome development in the tsetse fly vector. PLoS Pathog 14:e1007502. https://doi.org/10.1371/journal.ppat .1007502 .

38. Bursell E. 1981. The role of proline in energy metabolism. Plenum Press, New York, NY.

39. Turnock DC, Ferguson MA. 2007. Sugar nucleotide pools of Trypanosoma brucei, Trypanosoma cruzi, and Leishmania major. Eukaryot Cell 6:1450-1463. https://doi.org/10.1128/EC.00175-07.

40. Gualdron-Lopez M, Vapola MH, Miinalainen IJ, Hiltunen JK, Michels PA, Antonenkov VD. 2012. Channel-forming activities in the glycosomal fraction from the bloodstream form of Trypanosoma brucei. PLoS One 7: e34530. https://doi.org/10.1371/journal.pone.0034530.
41. Antonenkov VD, Rokka A, Sormunen RT, Benz R, Hiltunen JK. 2005. Solute traffic across mammalian peroxisomal membrane-single channel conductance monitoring reveals pore-forming activities in peroxisomes. Cell Mol Life Sci 62:2886-2895. https://doi.org/10.1007/s00018-005-5233-x.

42. Yernaux C, Fransen M, Brees C, Lorenzen S, Michels PA. 2006. Trypanosoma brucei glycosomal $\mathrm{ABC}$ transporters: identification and membrane targeting. Mol Membr Biol 23:157-172. https://doi.org/10.1080/09687860500460124.

43. Igoillo-Esteve M, Mazet M, Deumer G, Wallemacq P, Michels PA. 2011. Glycosomal ABC transporters of Trypanosoma brucei: characterisation of their expression, topology and substrate specificity. Int J Parasitol 41:429-438. https://doi.org/10.1016/j.ijpara.2010.11.002.

44. Colasante C, Voncken F, Manful T, Ruppert T, Tielens AG, Van Hellemond $\mathrm{JJ}$, Clayton C. 2013. Proteins and lipids of glycosomal membranes from Leishmania tarentolae and Trypanosoma brucei. F1000Res 2:27. https://doi .org/10.12688/f1000research.2-27.v1.

45. Kleczkowski LA, Kunz S, Wilczynska M. 2010. Mechanisms of UDP-glucose synthesis in plants. Crit Rev Plant Sci 29:191-203. https://doi.org/10.1080/ 07352689.2010 .483578$.

46. Colasante C, Ellis M, Ruppert T, Voncken F. 2006. Comparative proteomics of glycosomes from bloodstream form and procyclic culture form Trypanosoma brucei brucei. Proteomics 6:3275-3293. https://doi.org/10.1002/ pmic. 200500668 .

47. Guther ML, Urbaniak MD, Tavendale A, Prescott A, Ferguson MA. 2014. High-confidence glycosome proteome for procyclic form Trypanosoma brucei by epitope-tag organelle enrichment and SILAC proteomics. J Proteome Res 13:2796-2806. https://doi.org/10.1021/pr401209w.

48. Opperdoes FR, Szikora JP. 2006. In silico prediction of the glycosomal enzymes of Leishmania major and trypanosomes. Mol Biochem Parasitol 147:193-206. https://doi.org/10.1016/j.molbiopara.2006.02.010.

49. Chambers JK, Macdonald LE, Sarau HM, Ames RS, Freeman K, Foley JJ, Zhu Y, McLaughlin MM, Murdock P, McMillan L, Trill J, Swift A, Aiyar N, Taylor P, Vawter L, Naheed S, Szekeres P, Hervieu G, Scott C, Watson JM, Murphy AJ, Duzic E, Klein C, Bergsma DJ, Wilson S, Livi GP. 2000. A G protein-coupled receptor for UDP-glucose. J Biol Chem 275:10767-10771. https://doi.org/10.1074/jbc.275.15.10767.

50. Janse van Rensburg HC, Van den Ende W. 2017. UDP-glucose: a potential signaling molecule in plants? Front Plant Sci 8:2230. https://doi.org/10 .3389/fpls.2017.02230.

51. Thoms S. 2015. Import of proteins into peroxisomes: piggybacking to a new home away from home. Open Biol 5:150148. https://doi.org/10 .1098/rsob.150148.

52. Freitas MO, Francisco T, Rodrigues TA, Lismont $C$, Domingues $P$, Pinto MP, Grou CP, Fransen M, Azevedo JE. 2015. The peroxisomal protein import machinery displays a preference for monomeric substrates. Open Biol 5:140236. https://doi.org/10.1098/rsob.140236.

53. Yang J, Pieuchot L, Jedd G. 2018. Artificial import substrates reveal an omnivorous peroxisomal importomer. Traffic 19:786-797. https://doi.org/10 $.1111 /$ tra.12607.

54. Hunt M, Köhler P. 1995. Purification and characterization of phosphoenolpyruvate carboxykinase from Trypanosoma brucei. Biochim Biophys Acta 1249:15-22. https://doi.org/10.1016/0167-4838(95)00061-X.

55. Huybrechts SJ, Van Veldhoven PP, Brees C, Mannaerts GP, Los GV, Fransen M. 2009. Peroxisome dynamics in cultured mammalian cells. Traffic 10:1722-1733. https://doi.org/10.1111/j.1600-0854.2009.00970.x.

56. Delille HK, Agricola B, Guimaraes SC, Borta H, Luers GH, Fransen M, Schrader M. 2010. Pex11 pbeta-mediated growth and division of mammalian peroxisomes follows a maturation pathway. J Cell Sci 123:2750-2762. https://doi.org/10.1242/jcs.062109.

57. Costello JL, Schrader M. 2018. Unloosing the Gordian knot of peroxisome formation. Curr Opin Cell Biol 50:50-56. https://doi.org/10.1016/j.ceb .2018.02.002.

58. Lorenz P, Maier AG, Baumgart E, Erdmann R, Clayton C. 1998. Elongation and clustering of glycosomes in Trypanosoma brucei overexpressing the glycosomal Pex11p. EMBO J 17:3542-3555. https://doi.org/10.1093/ emboj/17.13.3542.

59. Hughes L, Borrett S, Towers K, Starborg T, Vaughan S. 2017. Patterns of organelle ontogeny through a cell cycle revealed by whole-cell reconstructions using 3D electron microscopy. J Cell Sci 130:637-647. https://doi .org/10.1242/jcs.198887.

60. Chanez AL, Hehl AB, Engstler M, Schneider A. 2006. Ablation of the single dynamin of $T$. brucei blocks mitochondrial fission and endocytosis and leads to a precise cytokinesis arrest. J Cell Sci 119:2968-2974. https://doi .org/10.1242/jcs.03023. 
61. Morgan GW, Goulding D, Field MC. 2004. The single dynamin-like protein of Trypanosoma brucei regulates mitochondrial division and is not required for endocytosis. J Biol Chem 279:10692-10701. https://doi.org/ 10.1074/jbc.M312178200.

62. Niemann M, Wiese S, Mani J, Chanfon A, Jackson C, Meisinger C, Warscheid B, Schneider A. 2013. Mitochondrial outer membrane proteome of Trypanosoma brucei reveals novel factors required to maintain mitochondrial morphology. Mol Cell Proteomics 12:515-528. https://doi .org/10.1074/mcp.M112.023093.

63. Veenhuis M, Sulter G, van der Klei I, Harder W. 1989. Evidence for functional heterogeneity among microbodies in yeasts. Arch Microbiol 151:105-110. https://doi.org/10.1007/BF00414422.

64. Tetley L, Vickerman K. 1991. The glycosomes of trypanosomes: number and distribution as revealed by electron spectroscopic imaging and 3-D reconstruction. J Microsc 162:83-90. https://doi.org/10.1111/j.1365-2818 .1991.tb03118.x.

65. Brun R, Schonenberger M. 1979. Cultivation and in vitro cloning or procyclic culture forms of Trypanosoma brucei in a semi-defined medium. Acta Trop 36:289-292.

66. Hirumi H, Hirumi K. 1989. Continuous cultivation of Trypanosoma brucei blood stream forms in a medium containing a low concentration of serum protein without feeder cell layers. J Parasitol 75:985-989. https://doi .org/10.2307/3282883.

67. Bringaud F, Baltz D, Baltz T. 1998. Functional and molecular characterization of a glycosomal PPi-dependent enzyme in trypanosomatids: pyruvate, phosphate dikinase. Proc Natl Acad Sci U S A 95:7963-7968. https:// doi.org/10.1073/pnas.95.14.7963.

68. Alibu VP, Storm L, Haile S, Clayton C, Horn D. 2005. A doubly inducible system for RNA interference and rapid RNAi plasmid construction in Trypanosoma brucei. Mol Biochem Parasitol 139:75-82. https://doi.org/10 .1016/j.molbiopara.2004.10.002.

69. Dean S, Sunter J, Wheeler RJ, Hodkinson I, Gluenz E, Gull K. 2015. A toolkit enabling efficient, scalable and reproducible gene tagging in trypanosomatids. Open Biol 5:140197. https://doi.org/10.1098/rsob.140197.

70. DeLoache WC, Russ ZN, Dueber JE. 2016. Towards repurposing the yeast peroxisome for compartmentalizing heterologous metabolic pathways. Nat Commun 7:11152. https://doi.org/10.1038/ncomms11152.

71. Opperdoes FR, Borst P. 1977. Localization of nine glycolytic enzymes in a microbody-like organelle in Trypanosoma brucei: the glycosome. FEBS Lett 80:360-364. https://doi.org/10.1016/0014-5793(77)80476-6.
72. Albisetti A, Florimond C, Landrein N, Vidilaseris K, Eggenspieler M, Lesigang J, Dong G, Robinson DR, Bonhivers M. 2017. Interaction between the flagellar pocket collar and the hook complex via a novel microtubule-binding protein in Trypanosoma brucei. PLoS Pathog 13:e1006710. https://doi.org/ 10.1371/journal.ppat.1006710.

73. Panicucci B, Gahura O, Zikova A. 2017. Trypanosoma brucei TbIF1 inhibits the essential F1-ATPase in the infectious form of the parasite. PLoS Negl Trop Dis 11:e0005552. https://doi.org/10.1371/journal.pntd.0005552.

74. Harlow E, Lane D. 1988. Antibodies: a laboratory manual. Cold Spring Harbor Laboratory Press, Cold Spring Harbor, NY.

75. Sambrook J, Fritsch EF, Maniatis T. 1989. Molecular cloning: a laboratory manual, 2nd ed. Cold Spring Harbor Laboratory Press, Cold Spring Harbor, NY.

76. Allmann S, Morand P, Ebikeme C, Gales L, Biran M, Hubert J, Brennand A, Mazet M, Franconi JM, Michels PA, Portais JC, Boshart M, Bringaud F. 2013. Cytosolic NADPH homeostasis in glucose-starved procyclic Trypanosoma brucei relies on malic enzyme and the pentose phosphate pathway fed by gluconeogenic flux. J Biol Chem 288:18494-18505. https://doi .org/10.1074/jbc.M113.462978.

77. Kralova I, Rigden DJ, Opperdoes FR, Michels PA. 2000. Glycerol kinase of Trypanosoma brucei. Cloning, molecular characterization and mutagenesis. Eur J Biochem 267:2323-2333. https://doi.org/10.1046/j.1432-1327 .2000.01238.x.

78. Pineda E, Thonnus M, Mazet M, Mourier A, Cahoreau E, Kulyk H, Dupuy JW, Biran M, Masante C, Allmann S, Riviere L, Rotureau B, Portais JC, Bringaud F. 2018. Glycerol supports growth of the Trypanosoma brucei bloodstream forms in the absence of glucose: analysis of metabolic adaptations on glycerol-rich conditions. PLoS Pathog 14:e1007412. https://doi .org/10.1371/journal.ppat.1007412.

79. Kall L, Canterbury JD, Weston J, Noble WS, MacCoss MJ. 2007. Semi-supervised learning for peptide identification from shotgun proteomics datasets. Nat Methods 4:923-925. https://doi.org/10.1038/nmeth1113.

80. Deutsch EW, Bandeira N, Sharma V, Perez-Riverol Y, Carver JJ, Kundu DJ, Garcia-Seisdedos D, Jarnuczak AF, Hewapathirana S, Pullman BS, Wertz J, Sun Z, Kawano S, Okuda S, Watanabe $Y$, Hermjakob $H$, MacLean B, MacCoss MJ, Zhu Y, Ishihama Y, Vizcaino JA. 2020. The ProteomeXchange consortium in 2020: enabling "big data" approaches in proteomics. Nucleic Acids Res 48:D1145-D1152. https://doi.org/10.1093/nar/gkz984. 